\title{
Il disegno della città rinascimentale dalle illustrazioni del De Nola ai dati cartografici contemporanei
}

\author{
Alessandra Avella \\ Nicola Pisacane \\ Pasquale Argenziano
}

Abstract

II paper presenta l'analisi della città cinquecentesca di Nola attraverso lo studio comparato delle quattro illustrazioni accluse al De Nola di Ambrogio Leone (15 |4), anche nel confronto con la città contemporanea. Tali disegni sono un'importante testimonianza grafico-documentale della città in quanto non solo restituiscono la morfologia dei luoghi al tempo della loro esecuzione ma anche per i metodi e le tecniche impiegati per la loro esecuzione, offrendosi, pertanto, a notevoli valutazioni di cui alcune sono raccolte nel presente saggio.

La ricerca partendo da analisi grafiche e geometriche e da stime metriche sui disegni presenti nel trattato si avvale degli approcci metodologici della scienza del Disegno non solo per una loro rilettura critica ma anche per connettere la città rinascimentale a tracce tuttora presenti nel tessuto urbano contemporaneo. II confronto con la contemporaneità non è teso solo alla determinazione delle permanenze nel tessuto viario ma anche ad alcune considerazioni sulle rappresentazioni di Nola a cura del Leone. Le quattro illustrazioni, infatti, si differenziano non solo per l'ambito spaziale in esse rappresentato, ma anche per i metodi di rappresentazione impiagati, talvolta per simulare la tridimensionalità della realtà orografica, in altri casi per offrire una verosimiglianza visiva alle architetture e al sistema di fortificazione della città a testimoniare un controllo generale del disegnatore sull'immagine della scena rappresentata.

Parole chiave

documentazione, geometria, trattatistica, GIS, UNESCO.

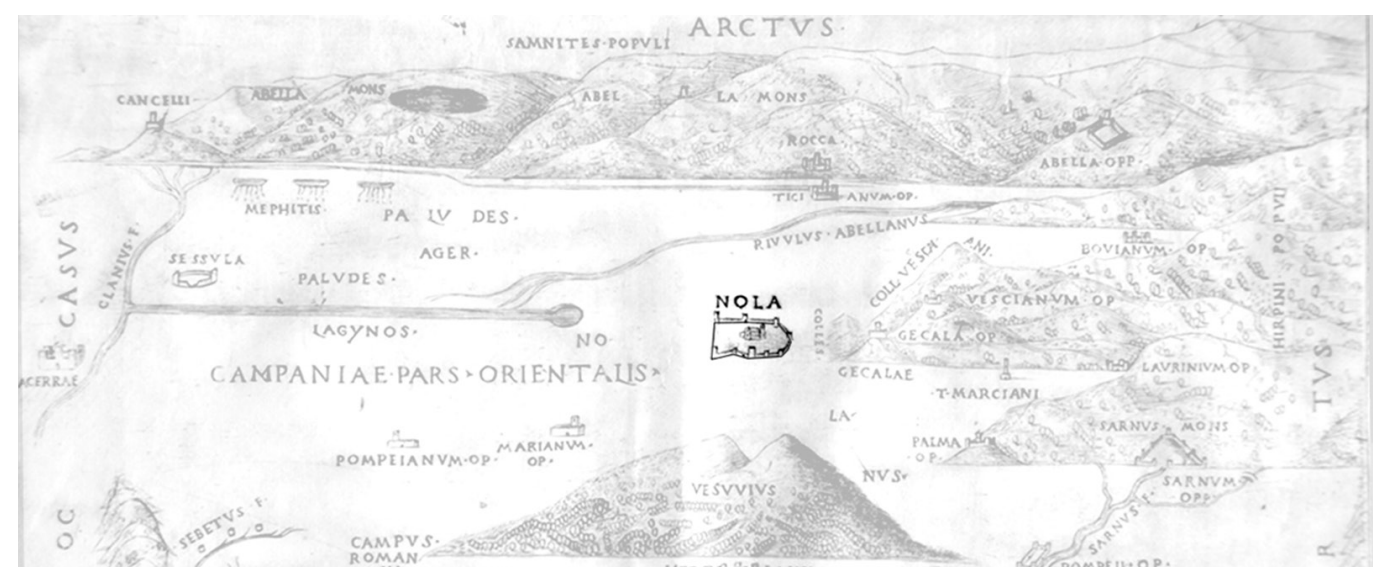




\section{Introduzione}

Il paper riporta i primi esiti delle ricerche condotte nell'ambito del Progetto di Ricerca finanziato dalla Regione Campania e finalizzato, in particolare, allo studio per la salvaguardia e valorizzazione degli elementi culturali dell'autenticità dei "Gigli di Nola", della sua festa e della sua comunità, iscritti nella lista del Patrimonio Immateriale UNESCO [I] In particolare, la ricerca affronta l'analisi dei quattro disegni allegati al volume De Nola di Ambrogio Leone [2] (I5I4) per i quali si propone una lettura inedita nel panorama degli studi, ampi e multidisciplinari editi nel corso degli ultimi decenni, approfondendo gli aspetti geometrici e grafici secondo un approccio metodologico proprio della disciplina del Disegno [3].Tralasciando la fortuna bibliografica che l'opera in latino ha avuto nel corso dei secoli [4], la ricerca propone la rilettura in chiave grafica delle ipotesi presentate nei saggi critici sulle iconografie dell'opera del Leone pubblicati dai primi del Novecento [5] fino a pochi anni orsono da studiosi attivi in vari campi della ricerca italiana.

L'analisi delle quattro illustrazioni (fig. I) è condotta su uno degli esemplari della prima edizione a stampa conservati nella Biblioteca Nazionale di Napoli [6]. Successivamente alla prima curata da Leone e verosimilmente da Mocetto [7], il De Nola vanta sei edizioni latine in volumi miscellanei o monografici e [8] due traduzioni in italiano [9].

Leone organizza l'opera in tre libri attraverso i quali in ordine descrive: il territorio nolano con taglio cronologico e con un'ampia dissertazione sulla città classica; la città a lui coeva e la sua struttura con approfondimenti sugli elementi difensivi, sulle emergenze architettoniche nobiliari, religiose e civili; e infine gli usi e i costumi dei suoi concittadini, dando così all'opera una nota di colore che raramente si nota in coeve omologhe pubblicazioni, tra i quali descrive anche la tradizione della Festa dei Gigli [ I 0], dal cui studio la presente ricerca trae spunto. L'edizione del 1514 edita a Venezia presso la stamperia di Giovanni Rosso da Vercelli, è composta da 64 carte a stampa tra le quali vi sono le quattro tavole fuori testo; queste, impresse su fogli diversi da quelli rilegati, sono state incollate alle pagine bianche atte ad accoglierli. La tavola Ager Nolanus (Tavola I) è posta a manca dell'inizio del Liber Primus (bks. I, I) [I I]; la tavola Nola Vetus (Tavola II) è alla pagina destra della chiusura del capitolo De incremento et potenti Nolanorum (bks. I, 6); la tavola Figura praesentis urbis Nolae (Tavola III) è posta a manca della conclusione del capitolo De figura et ambito praesentis (bks. II, 2); la tavola Nola Presens (Tavola IV) è posta sempre a manca dell'inizio del capitolo De muriis, pomoeriis [...] preasentis urbis (bks. II, 7) [I2].

Fig. I. Le quattro illustrazioni dal De Nola (edizione 15I4) oggetto di studio: (In alto a sinistra) Ager Nolanus (detta Tavola I), (in basso a sinistra) Nola Vetus (detta Tavola II); (in alto a destra) Figuro praesentis urbis Nolae (detta Tavola III): (in basso (destra) Nola), (in basso a destra) Nola presen

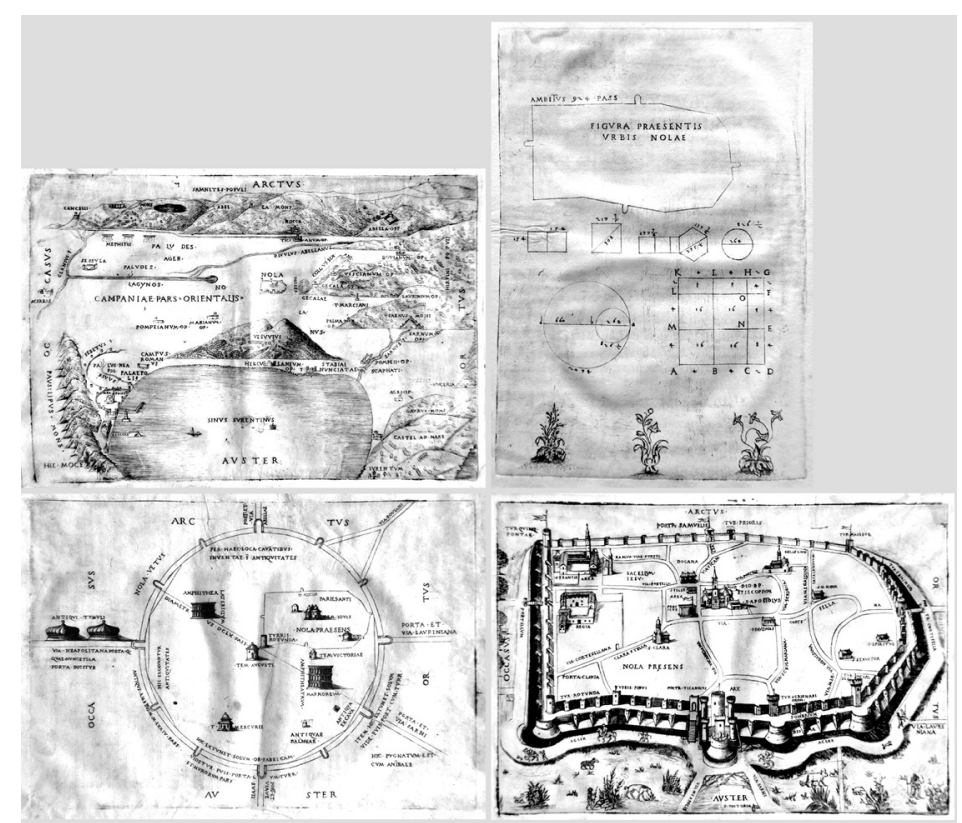


Le tavole, ad esclusione della Tavola III che presenta un carattere prevalentemente geometrico, hanno un comune impiego di differenti metodi di rappresentazione privilegiando una rappresentazione accidentale con un punto di vista in posizione elevata [Manzi 1973]. Nello specifico la Tavola I - l'unica tra le quattro firmata da Girolamo Mocetto - presenta un punto di vista dal golfo di Napoli verso nord con allineamento nella direzione della cinta muraria ovest della città di Nola.

L'allusione alla tridimensionalità è evidente dalla rappresentazione delle emergenze orografiche. La Tavola II appare come un'icnografia del tracciato murario della città romana lungo il quale la proiezione verticale delle 12 porte urbiche è rappresentata ribaltata nella direzione perpendicolare ai raggi. Le architetture rappresentate prevalentemente in proiezione verticale ortogonale appaiono iconiche delle rispettive tipologie piuttosto che allusive di una verosimiglianza con la realtà. La Tavola IV appare come focus scalare della Tavola I sulla città approfondendo quindi una rappresentazione verosimile dei luoghi visti dal Leone, sia del sistema difensivo che delle architetture, arricchendosi anche del disegno del tracciato viario. Le quattro Tavole presentano il comune allineamento della cinta occidentale della città con orientamento costante nord-sud, indipendentemente dal metodo di rappresentazione adottato e sempre parallelo al bordo verticale della tavola, quasi a voler essere il segno identificativo di Nola indipendentemente dalla scalarità della rappresentazione. Tale direzione giace lungo la mediana verticale delle Tavole I e II, mentre nella Tavola IV allinea la Cittadella fortificata con la Cattedrale.

Tutte le Tavole sono corredate da testi didascalici in caratteri capitali di dimensione variabile in relazione alla tipologia di informazione; fa ancora una volta eccezione la Tavola III nella quale il sistema di quotatura alfanumerico è in corsivo.

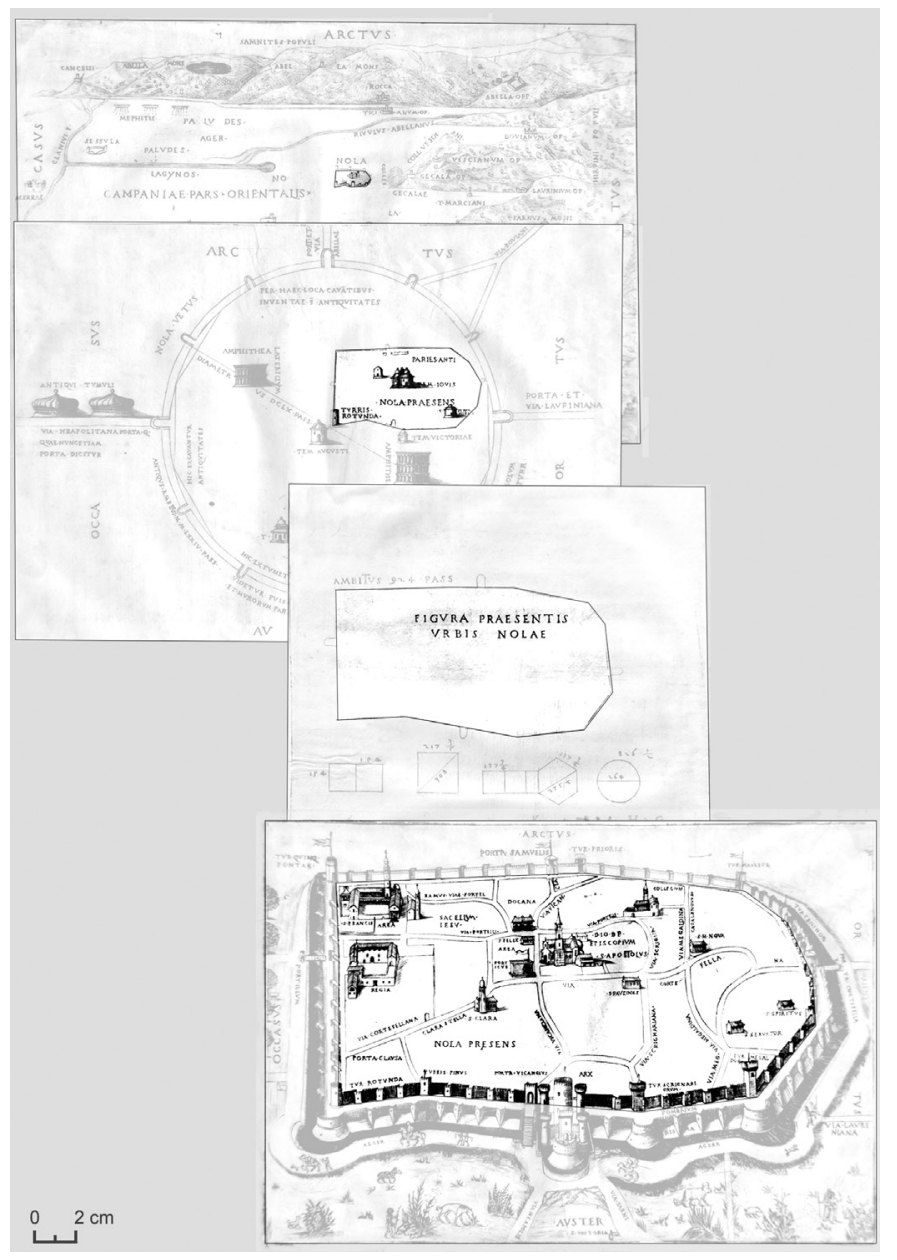




\section{Materiali, metodi e discussione}

Nella cornice della ricerca sulle iconografie del De Nola, rispettando questo testo si descrive l'iter investigativo dall'approccio metodologico alle sperimentazioni condotte sulle quattro illustrazioni che rappresentano la città rinascimentale nel contesto territoriale, a confronto della città romana, in riferimento alle sue dimensioni e in riferimento all'impianto urbano ed alle principali architetture in esso presenti. L'analisi è proseguita con valutazioni critiche inerenti i metodi di rappresentazione, i rapporti di forma e misura di ciascuna illustrazione e la forma urbis rinascimentale a confronto con i più aggiornati dati cartografici digitali su piattaforma GIS [I3].

E evidente che la ricerca si sia concentrata sulla produzione grafica del I 5 I 4 per rintracciare le intenzioni grafiche di Leone e di Mocetto, che molto probabilmente curarono l'edizione anche in fase di stampa, per porre solide basi critiche alla lettura comparata delle iconografie delle altre edizioni. Si rimanda a successive pubblicazioni la trattazione critica complessiva sul corpus iconografico attraverso le varie edizioni latine.

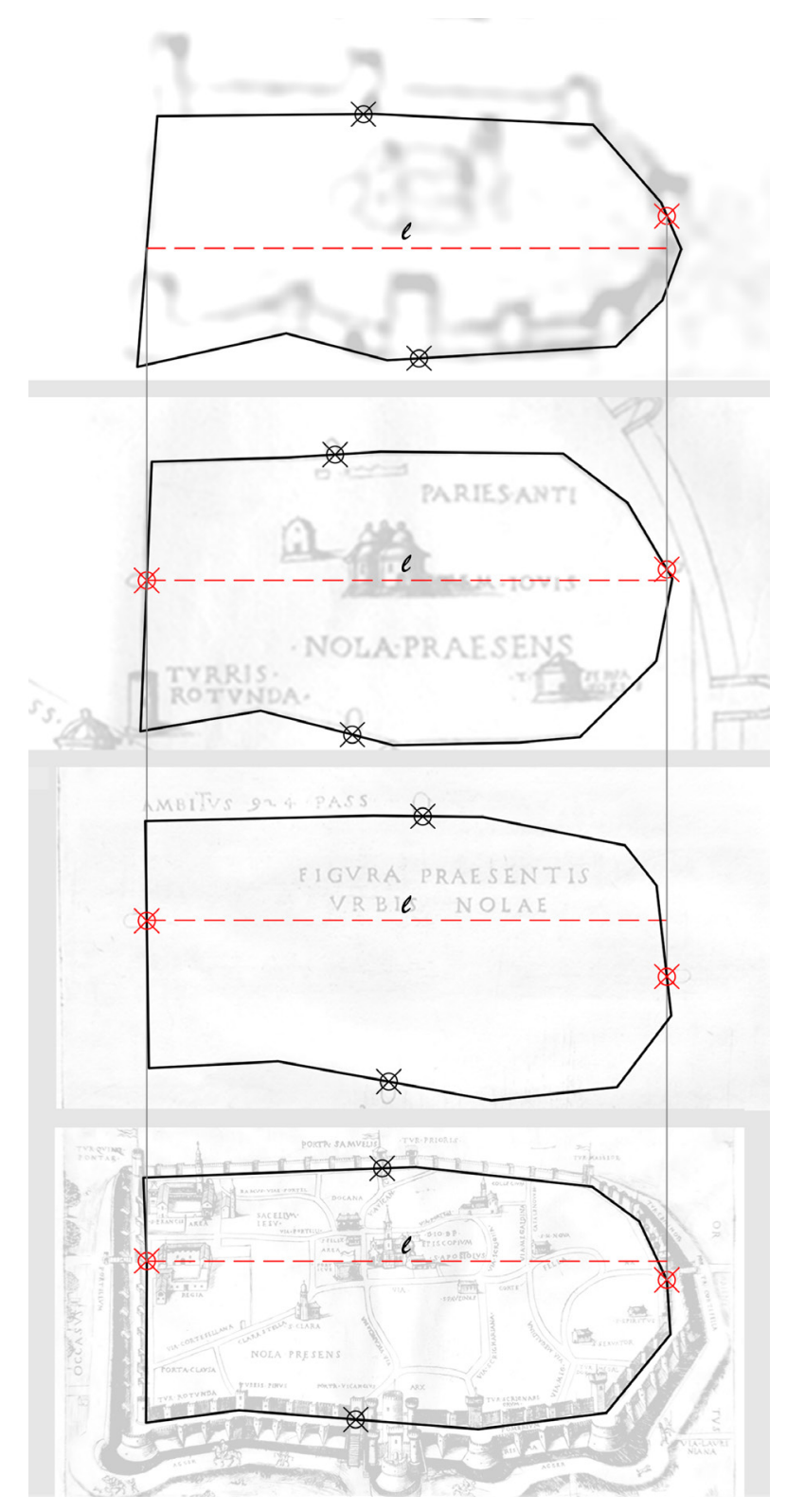


Fig. 4. Tavola III, individuazione e denominazione dei vertic e delle porte urbiche sulla poligonale della cinta muraria.

Fig. 5. Tavola IV individuazione $e$

denominazione dei vertici e delle porte urbiche sulla poligonale della cinta muraria.
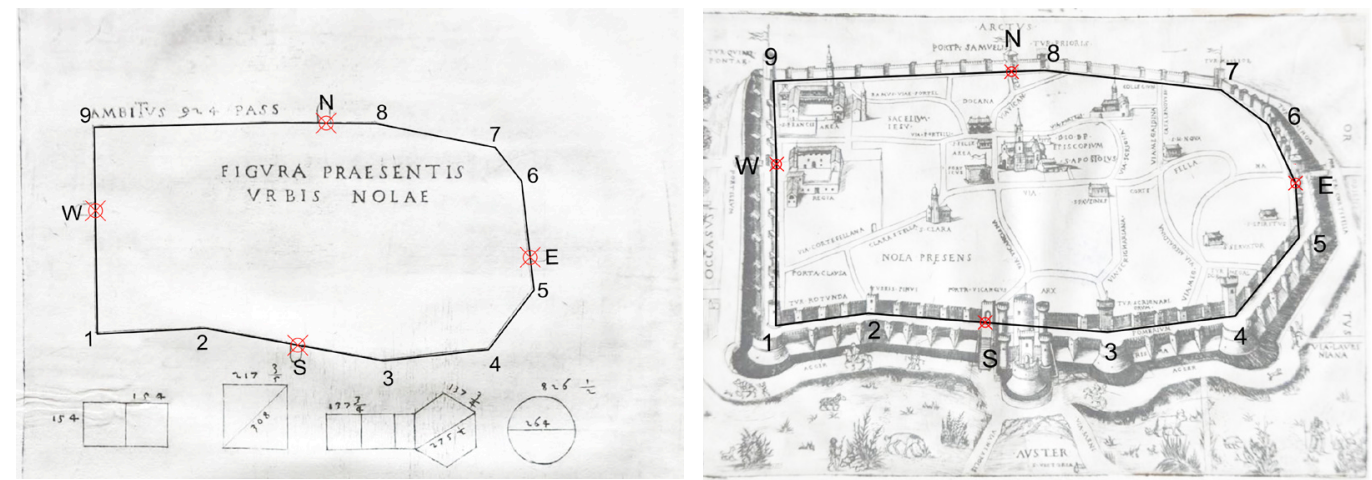

Le tavole allegate al volume consultato - come è evidente - hanno subito trasformazioni del supporto per effetto delle condizioni ambientali di conservazione e pertanto la consistenza fisica delle stesse non è esattamente paragonabile all'originale produzione. Per fissare la consistenza delle illustrazioni alla data di inizio della ricerca e per risolvere le aberrazioni prospettiche delle fotografie scattate a ridotta distanza dal soggetto, l'acquisizione delle immagini digitali è stata condotta accostando agli originali una scala graduata millimetrica e due scale cromatiche di quadricromia e di grigi; ed inoltre sono state prelevate le dimensioni dei supporti e delle cornici delle immagini.

Nel software RDF [14] le dimensioni dei fogli e delle immagini in essi impresse sono stati utilizzati per orto-rettificare e scalare al vero le fotografie [15]. Le scale cromatiche, solidali alle immagini orto-rettificate, sono state successivamente utilizzate per riprodurre la reale colorazione delle iconografie in uno dei più diffusi software di photo-editing. Secondo questa procedura, l'analisi grafica si fonda su 'modelli' fotografici in vera forma, grandezza e cromia delle iconografie del $|5| 4$.
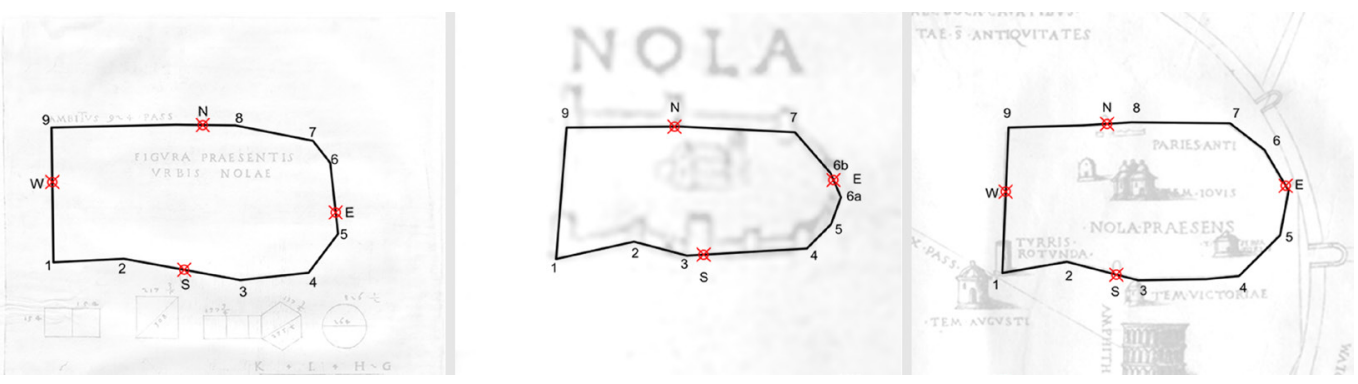

La lettura comparata delle quattro illustrazioni in scala (fig. 2) evidenzia che il perimetro, indicato da Leone come Figura praesentis urbis Nolae, è il segno ad esse comune e pertanto le successive analisi sono condotte a partire da questo elemento. Ad avvalorare questa scelta vi è l'ipotesi che Leone avesse realizzato un rilievo mensorio [De Divitiis, Lenzo, Miletti 20 I 8, p. 60] del perimetro urbano e che la Tavola III ne sia la restituzione grafica, anche per le valutazioni geometrico-dimensionali a confronto con la descrizione [Leone I5 |4, bks. II, 2]. Questo perimetro è tracciato da Leone nella Tavola II e indicato come Nola praesens, è ben distinguibile nella forma iconica disegnata nella Tavola I ed è riconducibile alla cinta muraria interna al pomerio della città così come disegnata nella Tavola IV.

La comparazione delle quattro illustrazioni, inoltre, evidenzia che a confronto con il perimetro della Tavola III quello disegnato nella Tavola I è una riduzione di circa il I $\%$, quello della Tavola II è una riduzione di circa il 50\%, mentre quello della Tavola IV è un ingrandimento del $170 \%$. 


\section{Dalle quattro illustrazioni del De Nola all'analisi grafico-geometrica del perimetro urbano}

L'impossibilità di proseguire il confronto tra le illustrazioni in ambiente raster, per le eccessive differenze tra le porzioni di immagini riconducibili al perimetro murario, ha indirizzato la ricerca verso il ridisegno vettoriale della icnografia urbana. Sui poligoni rappresentativi del perimetro urbano sono state intercettate le posizioni delle porte urbiche orientate secondo la descrizione del Leone rispetto alle quattro direzioni cardinali. Al fine di rendere confrontabili i quattro profili, che non risultano sovrapponibili, è stata assunta, come elemento di riferimento comune, la distanza est-ovest (linea base I) a partire dalla porta ovest, perché è l'unica ancora riconoscibile nel tessuto urbano attuale ed evidentemente rappresentata nelle Tavole II, III e IV. Invece, nella Tavola I la posizione della porta ovest è stata supposta lungo il segmento murario occidentale ad una distanza proporzionale alla media delle precedenti tre. La linea base I, essendo parallela ai quadri della rappresentazione, indipendentemente dal metodo di rappresentazione adottato, risulta confrontabile nelle quattro immagini. Pertanto, i quattro poligoni sono stati ad essa dimensionalmente scalati (fig. 3).

I successivi confronti assumono come immagine di riferimento la Tavola III poiché in riferimento a quanto già commentato sopra, il Leone offre una accurata descrizione dell'immagine anche con precisi riferimenti sia al perimetro che all'estensione della città. L'essenzialità dell'immagine consente di individuare i nove vertici della poligonale rappresentativi della città, oltre che le quattro porte, le cui proiezioni verticali appaiono graficamente ribaltate sul piano all'esterno del perimetro. La numerazione dei vertici riportata nella figura 4 è a partire dal vertice rappresentativo della torre difensiva sud-ovest in senso antiorario, secondo la più diffusa convenzione in geometria (fig. 4).

Fig. 7. Confronto tra il poligono del perimetro di cinta (in arancio) estratto dalla Tavola III, scalato rispetto alla lunghezza di 924 passi, e quello estratto (in blu) dalla Tavola IV. I due poligoni mantenendo fissa la

lunghezza della linea base I (distanza est-ovest a partire dalla porta ovest) sono allineati prima (in alto a sinistra) facendo coincidere i due poligoni nel vertice I, poi (al centro a sinistra) facendo coincidere le linee base dei due poligoni con orieine portici jure nel vertice ove uali spostamenti rispetto degli spostamenti rispetto ad un sistema cartesiano con origine nel punto I (in basso a sinistra). Nelle tabelle a destra, dall'alto in basso, calcolo: delle variazioni dimensionali della lunghezza dei singoli segmenti di cinta e degli angoli interni dei nove vertici, e delle variazioni di posizione dei singoli vertici espresse in passi.
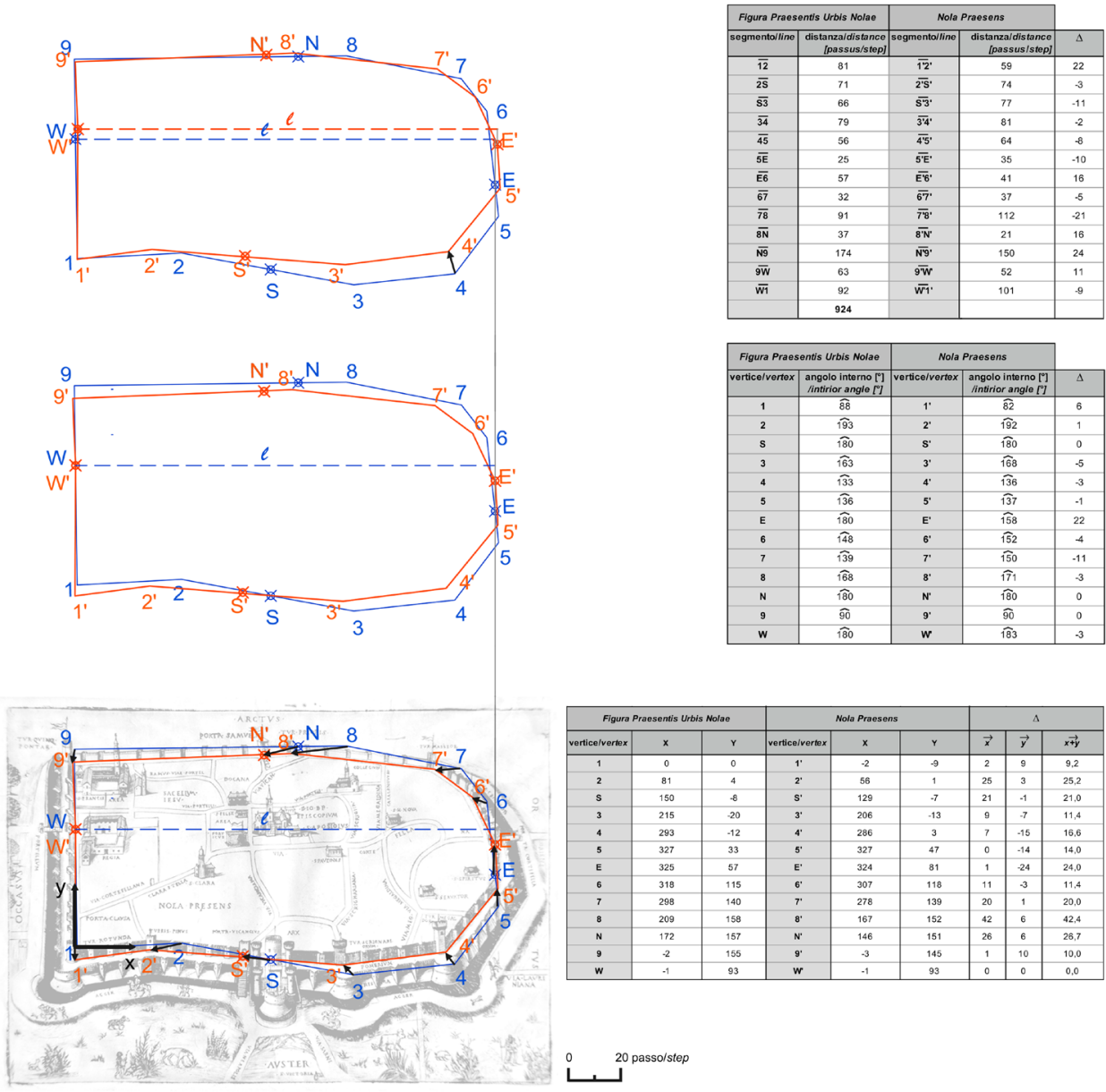
Gli omologhi vertici individuati nella Tavola IV (fig. 5), che corrispondono alle torri difensive della cinta muraria interna, sono chiaramente identificabili in una rappresentazione che simula la terza dimensione ed emergenti rispetto alla cresta della cinta. Nell'immagine in esame, tutte le nove torri, ad eccezione di quella presente nel vertice 5, sono indicate con la denominazione corrispondente. Tale denominazione è presente anche per le quattro porte di accesso alla città. Inoltre, la porta ad est è individuata su un ulteriore vertice della poligonale e non su un punto del segmento 5-6 come nellimmagine precedentemente commentata. L'immagine è rappresentata in un metodo allusivo della tridimensionalità poiché riporta lo sviluppo in quota nella rappresentazione sia della cinta muraria che di alcune architetture all'interno di essa, secondo una direzione prevalentemente verticale. Fa eccezione il tratto murario ad ovest, che presentando un forte scorciamento in profondità, induce il disegnatore a rappresentare le altezze secondo una direzione non verticale.

È possibile eseguire un simile confronto tra la Tavola III e le Tavole I e II. Dall'analisi comparativa emerge che sono sempre individuabili 9 vertici in ciascuna immagine. In particolare, però nella Tavola I, in cui la rappresentazione della città rinascimentale è inserita in un contesto territoriale molto più ampio, assunti e riconoscibili i vertici I e 7 nelle due immagini, si contano 8 vertici lungo tale tratto della poligonale. Diversamente nel tratto 7-9, il vertice 8 non risulta né riportato nella geometria del perimetro murario, né è identificabile attraverso il disegno di una torre, come invece è possibile per gli altri vertici. Le torri, nonostante la ridotta scala grafica di rappresentazione, che non restituisce un elevato dettaglio del disegno, sono rappresentate e riconoscibili nell'immagine. Tale semplificazione - come già precedentemente commentato - è operata anche per la porta ovest, dove l'orientamento della cinta muraria in riferimento al punto di vista, induce ad uno scorciamento che probabilmente il disegnatore risolve mancando la rappresentazione della suddetta porta.

Nella Tavola II, invece, la porta est, analogamente a quanto avviene nella Tavola IV, è individuata su un ulteriore vertice del poligono. Le quattro porte sono tutte rappresentate in proiezione verticale ribaltata esternamente al profilo per quelle ad ovest, nord ed est, ed internamente per quella a sud. L'immagine, inoltre, riproduce nel vertice I la rappresentazione di una torre a pianta circolare corrispondente al centro della circonferenza che, secondo il Leone, individua il perimetro della città romana. La più recente letteratura [De Divitiis, Lenzo, Miletti 2018, pp. 70] avanza l'ipotesi che il rilevatore si pose su questa torre angolare per eseguire il rilevamento topografico evidentemente da uno dei punti eminenti sull'abitato (fig. 6).

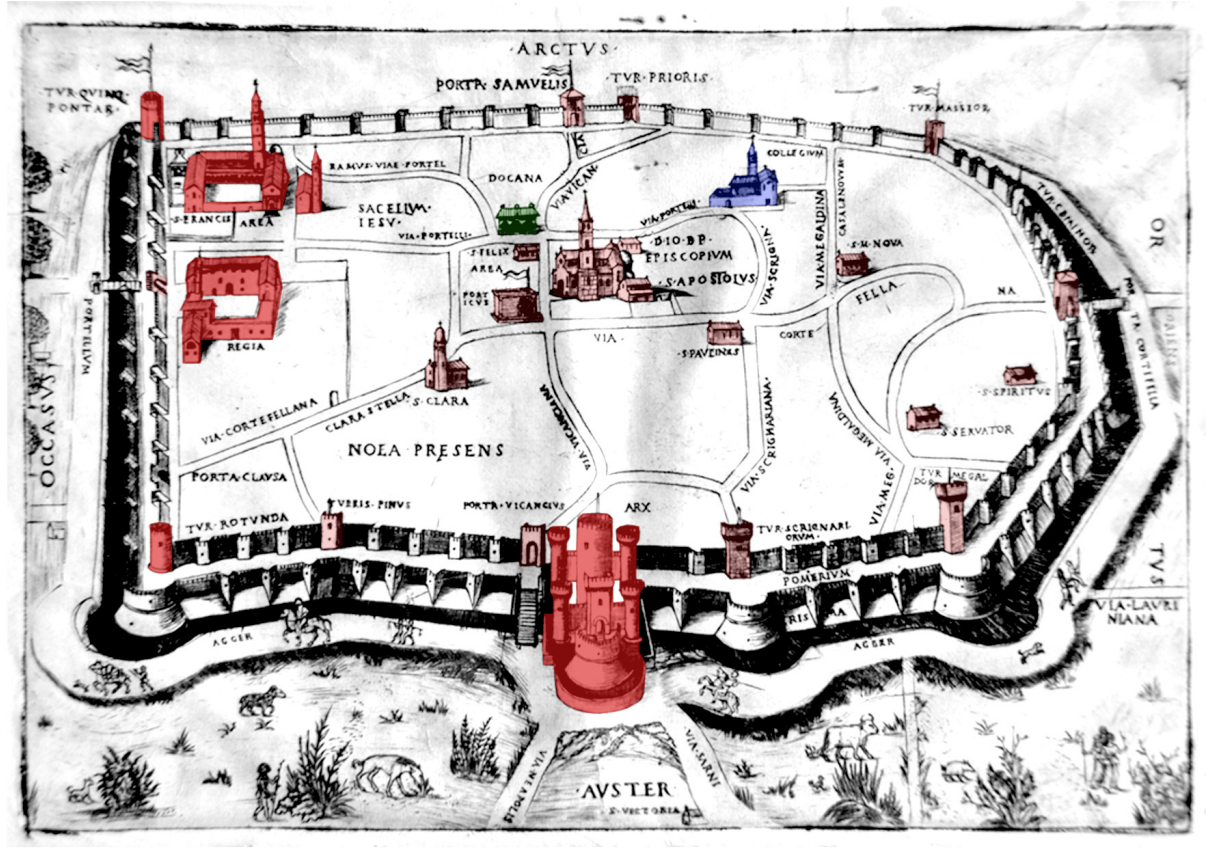




\section{Dalle valutazioni metrico-dimensionali alle ipotesi sui metodi di rappresentazione nell'illustrazione Nola Presens}

L'analisi prosegue approfondendo il confronto tra le Tavole III e IV, le più significative e dettagliate non solo ai fini di uno studio della città rinascimentale, ma anche in merito ai metodi di rappresentazione adottati nelle illustrazioni in esame. II poligono estratto dalla Tavola III viene scalato al fine di valutare l'effettiva lunghezza dei singoli segmenti di cinta intercorrenti tra le torri e tra le torri e le porte. Tale operazione viene riferita all'unico dato metrico riportato dallo stesso Leone e corrispondente a 924 passi.

La consistenza dimensionale è l'elemento di confronto con la Tavola IV i cui valori determinano il calcolo delle variazioni dimensionali dei succitati segmenti. Analoga valutazione è proposta per gli angoli interni dei 9 vertici del poligono e dei quattro punti rappresentativi delle porte. Anche in tal caso la valutazione della differenza angolare è opportunità di valutazioni che di seguito si riportano.

Come già anticipato nella trattazione, i perimetri delle immagini non sono sovrapponibili ma una più approfondita disamina dei poligoni viene condotta secondo due ipotesi. La prima, sempre mantenendo fissa la lunghezza della linea base I - calcolata nella dimensione di 326 passi - fa coincidere i due poligoni nel vertice I allineandoli secondo il segmento I-9. La seconda, invece, fa coincidere le linee base dei due poligoni con origine nel vertice ovest. II confronto viene operato riferendo le posizioni dei vertici rispetto ad una coppia di assi ortogonali orientati con origine sempre nel punto I. La scelta di tale punto fa riferimento all'ipotesi sopra riportata di probabile punto di stazione del rilevamento mensorio e anche al fine di avere valori delle coordinate quasi tutti positivi. II vettore spostamento viene anche in questo caso calcolato per i tredici vertici, di cui nove riferiti alle torri e quattro alle porte, riferendolo tanto alla direzione delle ascisse e delle ordinate, quanto del vettore somma. L'analisi di tali valori evidenzia che nella prima ipotesi i perimetri, se sono pressoché sovrapponibili per l'intero tracciato tra i vertici 2 ed E, presentano, invece, una rigida traslazione di I 8 passi circa nei vertici 3 e 4 che fa supporre che il disegnatore potrebbe aver deformato il profilo per assecondare una vista pseudo-assonometrica vista da sud-est, secondo una direzione obliqua del centro di vista. La seconda ipotesi, invece, è quella che individua variazioni di posizione dei vertici tali che:

Fig. 9. Georeferenziazione in ambiente GIS dei punti caratteristici dell'impianto viario presenti nella Tavola IV e tuttora riconoscibili nel tracciato contemporaneo Individuazione poraneo. Individuazione de 30 punti sularto e n. 30 punti su ortofoto attuale (in alto a sinistra) individuazione dei punti omologhi sulla Tavola IV e indicazione dei vettor degli spostamenti (in alto a destra) e tabella di riepilogo delle coordinate in pixel dell immagine matrice e quelle cartografiche (rispetto al sistema cartografico WGS84/ UTM 33N) dell'omologo punto georiferito e dei relativi spostamenti (in basso).
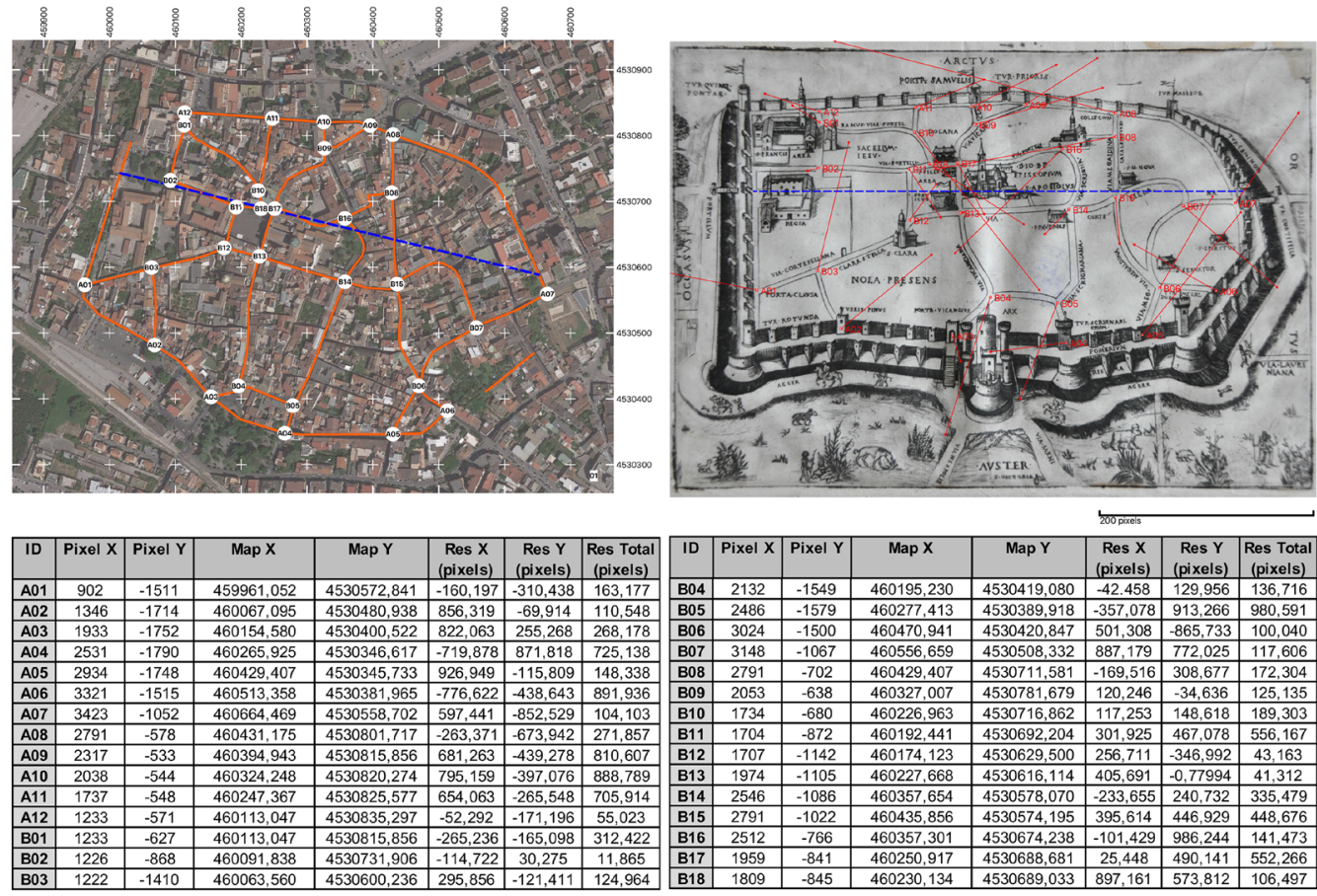
- laddove la variazione dello spostamento lungo la direzione delle ascisse è maggiore, risulta minimo il valore dello spostamento lungo la direzione delle ordinate; e viceversa.

- i vertici 9, N, 8 e 3, 4, rispettivamente appartenenti al tratto settentrionale e meridionale della cinta, presentano lo spostamento minore secondo la direzione verticale e pressoché costante, oltre che inferiore, rispetto a quello di tutti gli altri.

- gli spostamenti letti in valore assoluto, ad eccezione del vertice 8, hanno una variazione tra 9,2 e 26,7 passi (fig. 7).

Le succitate considerazioni fanno presupporre che l'immagine della Tavola IV sia rappresentata in un metodo pseudo-assonometrico, non solo per l'evidente disegno degli elementi architettonici in elevato della scena urbana, ma anche per la costante riduzione delle misure in profondità, che fanno supporre una posizione ortogonale del centro di proiezione assonometrica come evidentemente si osserva dall'immagine del Castello, della Reggia e dell'area di San Francesco.
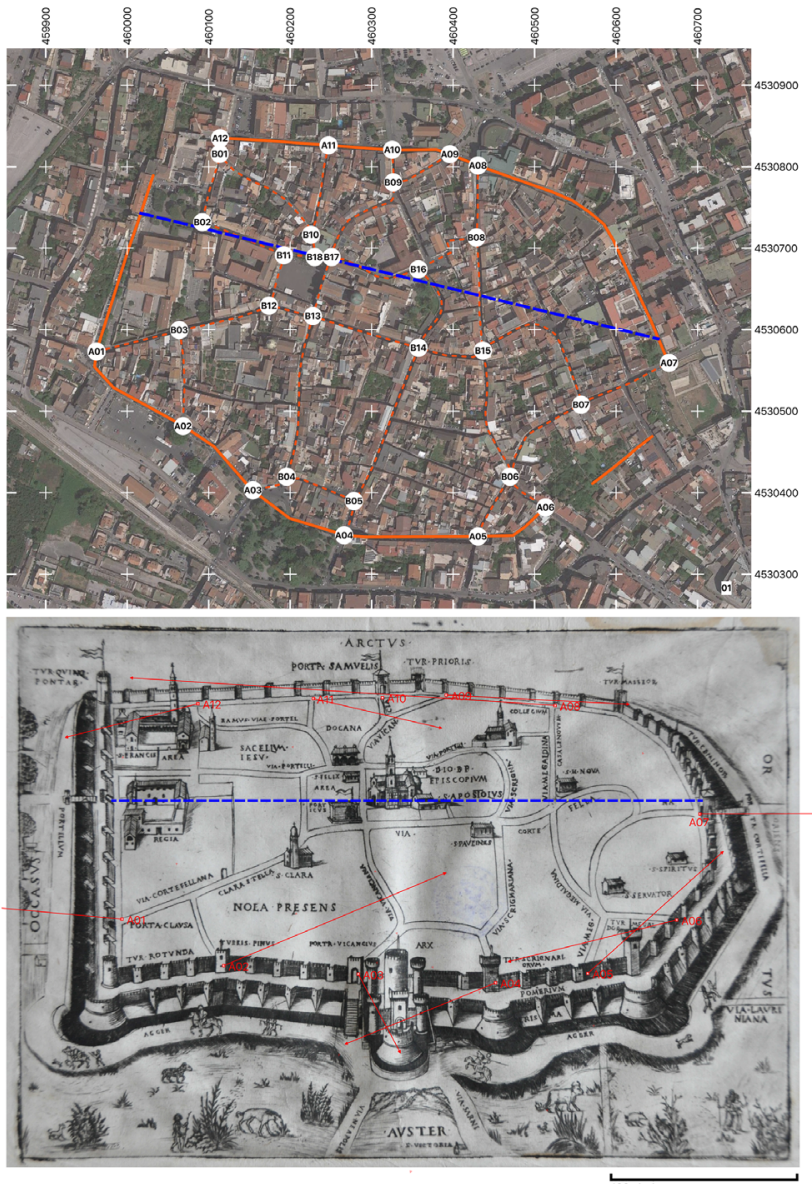

\begin{tabular}{|l|c|c|c|c|c|c|c|}
\hline ID & Pixel X & Pixel Y & Map X & Map Y & $\begin{array}{c}\text { Res X } \\
\text { (pixels) }\end{array}$ & $\begin{array}{c}\text { Res Y } \\
\text { (pixels) }\end{array}$ & $\begin{array}{c}\text { Res Total } \\
\text { (pixels) }\end{array}$ \\
\hline A01 & 902 & -1511 & 459961,052 & 4530572,841 & $-110,707$ & $-101,244$ & 111,169 \\
\hline A02 & 1346 & -1714 & 460067,095 & 4530480,938 & 118,952 & $-497,587$ & 128,94 \\
\hline A03 & 1933 & -1752 & 460154,58 & 4530400,522 & 225,432 & 421,825 & 478,284 \\
\hline A04 & 2531 & -1790 & 460265,925 & 4530346,617 & $-80,483$ & 328,623 & 869,336 \\
\hline A05 & 2934 & -1748 & 460429,407 & 4530345,733 & 725,372 & $-65,407$ & 976,715 \\
\hline A06 & 3321 & -1515 & 460513,358 & 4530381,965 & $-901,669$ & 223,516 & 92,896 \\
\hline A07 & 3423 & -1052 & 460664,469 & 4530558,702 & 961,215 & $-0,22677$ & 961,218 \\
\hline A08 & 2791 & -578 & 460431,175 & 4530801,717 & $-227,33$ & $-148,496$ & 227,814 \\
\hline A09 & 2317 & -533 & 460394,943 & 4530815,856 & 979,477 & 491,833 & 980,711 \\
\hline A10 & 2038 & -544 & 460324,248 & 4530820,274 & 964,157 & 628,821 & 966,205 \\
\hline A11 & 1737 & -548 & 460247,367 & 4530825,577 & 686,935 & 159,102 & 705,119 \\
\hline A12 & 1233 & -571 & 460113,047 & 4530835,297 & $-709,393$ & 180,915 & 732,098 \\
\hline
\end{tabular}




\section{Permanenze della città rinascimentale nel tessuto urbano contemporaneo}

Elemento comune all'intera scena è offerto dalla rappresentazione allusiva della tridimensionalità. II disegnatore associa a una vista frontale lo scorcio laterale tipico dell'impostazione della prospettiva frontale di difficile controllo per la scena oggetto della rappresentazione. Infatti, si può notare che da ovest ad est lo scorcio degli edifici e di alcune sezioni della cinta muraria si mantiene parallelo per settori, variando la direzione delle grandezze in profondità. Tale artificio grafico sembra quasi simulare il dinamismo del centro di proiezione. Si può quindi ipotizzare che piuttosto che una rappresentazione in pseudo-prospettiva, l'immagine combini plurime viste assonometriche. Ad un'impostazione che testimonia il controllo generale del disegnatore sulla scena rappresentata, è da evidenziare il disegno della cinta occidentale con orientamento fortemente scorciato, che è risolto con una costante rotazione della direzione degli spigoli verticali. Tale evidente anomalia grafica stride con il corretto disegno delle altezze dei torrioni e dei baloardetti [16] nel tratto 4'-7' (dalla Torre Megaldor alla Torre Masseor).

L'analisi è stata successivamente approfondita classificando le architetture rappresentate all'interno della cinta muraria e le emergenze turrite di quest'ultima secondo l'assonanza formale ai metodi di rappresentazione (proiezioni parallele e centrali) ad ulteriore conferma di un punto di vista dinamico. La maggior parte delle architetture poste ad occidente appaiono rappresentate in pseudo-assonometria ortogonale, diversamente da quelle disegnate nella restante parte della città che alludono ad un uso della pseudo-assonometria obliqua. Fa eccezione la rappresentazione dell'edificio indicato come Collegium che appare in prospettiva frontale (fig. 8).

I ridotti scorciamenti delle misure in profondità tra il tracciato riportato nella Tavola ||| e quello della Tavola IV precedentemente commentati (fig. 7) fanno ipotizzare che anche il disegno del tracciato viario all'interno della cinta muraria riprodotto nella Tavola IV possa essere esito di un rilevamento mensorio. Assumendo per vera questa ipotesi, si è proceduto alla georeferenziazione in ambiente GIS dei punti caratteristici dell'impianto viario tuttora riconoscibili nel tracciato contemporaneo. Nota la risoluzione dell'immagine orto-rettificata, essa è stata scalata rispetto alla dimensione in passi della linea base I (326 passi) corrispondente a 2580 pixel. Pertanto il pixel ha dimensione di 0, 13 passi.

Non essendo riconoscibili i 9 torrioni e le porte, ad esclusione di quella ovest, lungo la cinta muraria sono stati identificati i 12 punti di intersezione tra la strada perimetrale la cinta stessa e le strade ad essa convergenti. Pertanto, la georeferenziazione è stata dapprima eseguita attraverso 30 punti omologhi, di cui i restanti 18 sono esemplificativi degli incroci stradali ancora riconoscibili nel confronto tra il disegno e l'ortofoto attuale (fig. 9). Successivamente analoga operazione è stata condotta attraverso i soli 12 vertici lungo la cinta (fig. 10). Tralasciando l'esito della deformazione grafica dell'immagine matrice (Tavola IV) che produce una notevole variazione della forma che sminuisce valutazioni di carattere numerico, la lettura critica della georeferenziazione può essere fatta stimando le variazioni degli spostamenti tra le coordinate in pixel dell'immagine matrice e quelle cartografiche dell'omologo punto georiferito. Tali valori espressi in pixel e quindi riconducibili alle dimensioni in passi, con riferimento alla succitata corrispondenza dimensionale, evidenziano valori di scostamento maggiori per i punti interni esemplificativi degli incroci stradali rispetto a quelli sul perimetro murario.

\section{Conclusioni}

Le evidenze dell'analisi comparativa, esito della georeferenziazione, awalorano la considerazione che il disegnatore abbia elaborato la Tavola IV a partire dal disegno del perimetro murario in seguito a un rilevamento mensorio, piuttosto che dalla completa planimetria rappresentativa del tracciato viario e delle architetture eminenti nel tessuto urbano, che tra l'altro non trova riscontro in nessuna tavola. Non a caso l'unico riferimento dimensionale riportato nell'opera dal Leone è relativo al perimetro di 924 passi della cinta muraria. 
Un'ulteriore valutazione, riferita al confronto sopra descritto, è relativa alla posizione e all'orientamento della linea base I all'esito della georeferenziazione. Tale segmento, mentre nelle tavole del Leone ha un costante orientamento $90^{\circ} \mathrm{N}$, nella reale topografia risulta ruotato di $14^{\circ}$ rispetto al precedente. È facile dedurre che Leone abbia forzato la disposizione planimetrica delle porte urbiche rispetto all'orientamento dei margini dell'illustrazione che fa coincidere erroneamente con l'orientamento cardinale.

Tali risultati aprono ad ulteriori ed inesplorati possibili scenari investigativi che, a partire da considerazioni supportate dall'applicazioni grafiche di strumenti e metodi propri del Disegno, potranno fornire ulteriori elementi utili a valutare se le rappresentazioni siano dawero restituzioni di un rilevamento mensorio e quindi esito di un'avvenuta attività di acquisizione del dato metrico, come riportato in letteratura. In caso positivo, la ricerca approfondirà come tali operazioni di rilevamento siano state condotte e con quali strumenti in uso al tempo di Leone, oltre che a stimare la precisione della restituzione a partire dai dati acquisiti. A tal uopo, gli strumenti GIS, già valutati come idonei per le operazioni geometriche di comparazione sopra descritte, potranno confermarsi come utile ausilio per le ulteriori analisi che si propongono in merito al calcolo del possibile errore di acquisizione del dato e di confronto tra le unità di misura impiegate dal Leone con i sistemi metrici odierni [17].

\section{Note}

[ I] I Gigli di Nola rientrano, unitamente alla "Nostra Signora della Santa Lettera" di Palmi, alla "Discesa dei Candelieri" di Sassari e alla "Macchina di Santa Rosa" di Viterbo, nella "Rete delle grandi macchine a spalla italiane", iscritta dal 2013 nella Lista del Patrimonio Culturale Immateriale dell'UNESCO.

[2] Ambrogio Leone (Nola | 458 ca - Venezia 1525) è una singolare figura della cultura italiana rinascimentale, di collegamento tra gli ambienti degli eruditi napoletani, quelli veneziani e quelli d'oltralpe che frequentavano le rispettive capitali. A 26 anni consegue il dottorato in medicina e filosofia a Padova e tornato a Nola esercita la professione medica nella sua casa fuori città, che diviene luogo di ritrovo, anche per la felice cornice agreste, di eminenti uomini di cultura tra i quali Giovanni Pontano, noto e influente umanista e uomo politico, e il milanese Aurelio Bienato, lettore di retorica all'Università di Napoli. All'inizio del nuovo secolo, Leone si sposta prima a Padova e poi a Venezia esercitando la sua professione nel sestiere di San Marco e tornando saltuariamente a Nola fino all'alluvione del 1504 che segnò profondamente le sorti della città campana. A Venezia ebbe modo di distinguersi tra le cerchie della cultura lagunare, tant'è che ebbe modo di stringere rapporti di amicizia con Aldo Manuzio, entrando nel suo circolo di eruditi, noto come "dei Filelleni". Aspetto questo di particolare rilievo per la collocazione culturale e le valutazioni grafiche del De Nola. La frequentazione assidua di questa cerchia culturale, lo portò a conoscere Erasmo da Rotterdam con il quale rimase in contatto oltre il periodo di residenza dell'olandese in Laguna, come documentato da due lettere tra loro intercorse tra il 1518 e il ' 19.

Nel gennaio 1510 , le celebrazioni funebri di Niccolò Orsini - conte di Nola e capitano generale delle truppe veneziano nella guerra della Lega di Cambrai (1508-1516) - furono nodali per la realizzazione del De Nola, perché in quell'occasione ebbe certamente modo di conoscere (qualora non l'avesse fatto in precedenza) Girolamo Mocetto. Leone partecipò al funerale perché nolano e amico della Famiglia Orsini, Mocetto in quel momento vive a Venezia ed era attivo nella chiesa domenicana dei SS. Giovanni e Paolo di Venezia perché impegnato nella decorazione della vetrata dipinta del transetto destro, nella quale inserirà in segno di commemorazione, tra gli altri, la figura del condottiero Orsini [Spruit 2005].

[3] Si veda:Vagnetti 1973; Pagnano 2003; Zerlenga 2004.

[4] Si veda: Carillo 1996;Valerio 1998; De Divitiis, Lenzo, Miletti 2018.

[5] Nell'economia di questo testo non è possibile enumerare tutte le pubblicazioni edite e consultate, rimandando ai agli specifici riferimenti nelle altre note. La rassegna della completa bibliografia è in: De Divitiis, Lenzo, Miletti 20 I8.

[6] La collocazione dell'esemplare è SQ.LVI.D. 16

[7] Discendente di una famiglia di vetrai, Girolamo Mocetto (Murano | $470 \mathrm{ca} \mathrm{-} \mathrm{|53|} \mathrm{ca)} \mathrm{è} \mathrm{più} \mathrm{attivo} \mathrm{nella} \mathrm{pittura} \mathrm{e} \mathrm{nell'incisione,}$ rispetto alla tradizione familiare. Sebbene sulla sua formazione artistica non vi siano notizie certe, le tre possibilità note ben lo qualificano: nelle Vite, il Vasari lo indica come allievo di Giovanni Bellini; in tempi più recenti, altri protendono per la frequentazione della bottega di Alvise Vivarini in Murano oppure per l'appartenenza alla cerchia di Andrea Mantegna, dal quale avrebbe appreso l'arte incisoria a bulino. Tagliaferro (20 I I).

[8] De Divitiis, Lenzo, Miletti 20 8, p. 5.

[9] Leone 1934; Leone 1997.

[10] Leone I5I4, bks. III, 7.

[1 I] L'abbreviazione indica il riferimento al libro in numerazione cardinale e ai capitoli dell'opera in numerazione araba (ISO 690: 1987).

[ 2 2] || catalogo dei disegni pubblicati durante il XVIII secolo è in: de Seta, Buccaro 2006, pp. 273, 274

[13] La ricerca sui disegni del De Nola integra le conoscenze sull'iconografia vedutistica e sulla cartografia del Cinquecento. Le più recenti pubblicazioni consultate su questi temi sono: De Seta Cesare, Buccaro, Alfredo 2006; il numero monografico della rivista DisegnareCon sul tema Drawing the Territory and the Landscape (vol. I2 no.22, 20 I 9) a cura di Pilar Chìs Navarro 
e Lia Maria Papa; in questa rivista si segnala Aguilar-Camacho, Joaquín, Granado-Castro, Gabriel and Barrera-Vera, José Antonio, (2019). Urban mapping of Cadiz during the War of Spanish Succession: a paradigm of the cartography enterprise undertaken by the Military Engineer Corps. DisegnareCon. 2019. Vol. 12, no. 22, p. I- I3

[ | 4$]$ || software RDF è liberamente utilizzabile per gentile concessione del Laboratorio di Fotogrammetria CIRCE dell'Università IUAV di Venezia: <http://www.iuav.it/SISTEMA-DE/Laboratori2/cosa-offri/software/index.htm>.

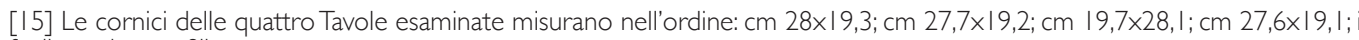
fogli non hanno filigrana.

[16] Contrafforti prismatici di rinforzo nel tratto della cinta muraria tra due torrioni: Sardi I6I 8, p. 40.

[17] Gli autori hanno progettato la ricerca e condiviso la sua metodologia e i suoi contenuti. In particolare, il paragrafo "Dalle quattro illustrazioni del De Nola all'analisi grafico-geometrica del perimetro urbano" è curato da Pasquale Argenziano, i paragrafo "Dalle valutazioni metrico-dimensionali alle ipotesi sui metodi di rappresentazione nell'illustrazione Nola Presens" è curato da Alessandra Avella e il paragrafo "Permanenze della città rinascimentale nel tessuto urbano contemporaneo" è curato da Nicola Pisacane. L'introduzione e le conclusioni sono curate da tutti gli autori. Le immagini a corredo del testo, ad eccezione di quelle di repertorio, sono a cura degli autori.

\section{Riferimenti bibliografici}

Carillo Saverio (1996). Alcune osservazioni sulla pianta della città allegata al De Nola di Ambrogio Leone. In:Toscano Tobia R. Nola e il suo territorio dalla fine del Medio Evo al XVII secolo. Momenti di storia culturale e artistica. Castellammare di Stabia:Ager Nolanus. p. 25-44.

De Divitiis Bianca, Lenzo Fulvio, Miletti Lorenzo (a cura di). (2018). Ambrogio Leone's De Nola, Venice 15/4. Humanism and Antiquarian Culture in Renaissance Southern Italy. Leiden, Boston: Brill.

De Seta Cesare, Buccaro Alfredo (a cura di). (2006). Iconografia delle città in Campania. Napoli e i centri della provincia. Napoli: Electa Napoli.

Leone Ambrogio (15|4). De Nola. Opusculum distinctum, plenum, clarum, doctum, pulchrum, verum, grave, varium, et utile. Venezia: Giovanni Rosso

Leone Ambrogio (1934). Nola (la terra natia). Napoli:Tipografia Torella.

Leone Ambrogio (1997). Nola. Napoli; Istituto Grafico Editoriale Italiano.

Manzi Pietro (1973). Alcuni documenti di cartografia nolana ovvero: Ambrogio Leone e Gerolamo Moceto. In L'Universo. Vol. LIII, no. 4, p. $81|-8| 8$.

Pagnano Giuseppe (2003). Presentazione. In Ikhnos. Analisi grafica e storia della rappresentazione. Vol. I, p. 7- I 0.

Sardi Pietro (16|8). Corona imperiale dell' architettura militare. Venezia: Barezzo Barezzi.

Spriut Leendert (2005). Leone, Ambrigio. In Dizionario Biografico degli Italiani, ad vocem. Treccani: <http://www.treccani.it/ enciclopedia/ambrogio-leone_(Dizionario-Biografico)/>.

Tagliaferro Giorgio, Mocetto Girolamo (20 I I). In Dizionario Biografico degli Italiani. Ad vocem. Treccani.

Vagnetti Luigi (1973). L'architetto nella storia di Occidente. Firenze:Teorema.

Valerio Vladimiro (1998). Piante e vedute di Napoli dal 1486 al 1599. L'origine dell'iconografia urbana europea. Napoli: Electa Napoli.

Zerlenga Ornella (2004). II disegno della città. Napoli rappresentata in Pianta e Veduta. In Ikhnos. Analisi grafica e storia della rappresentazione.Vol. II, p. I |-34.

\section{Autori}

Alessandra Avella, Università della Campania “Luigi Vanvitelli”, alessandra.avella@unicampania.it,

Nicola Pisacane, Università della Campania “Luigi Vanvitelli", nicola.pisacane@unicampania.it,

Pasquale Argenziano, Università della Campania "Luigi Vanvitelli", pasquale.argenziano@unicampania.it

Per citare questo articolo: Avella Alessandra, Pisacane Nicola, Argenziano Pasquale (2020). Il disegno della città rinascimentale dalle illustrazioni del De Nola ai dati cartografici contemporanei/The Drawing of the Renaissance city from De Nola's tables to contemporary cartographical data. In Arena A., Arena M., Brandolino R.G., Colistra D., Ginex G., Mediati D., Nucifora S., Raffa P. (a cura di). Connettere. Un disegno per annodare e tessere Atti del $42^{\circ}$ Convegno Internazionale dei Docenti delle Discipline della Rappresentazione/Connecting. Drawing for weaving relationships. Proceedings of the 42th International Conference of Representation Disciplines Teachers. Milano: FrancoAngeli, pp. I 598-I 621 . 


\title{
The Drawing of the Renaissance City from De Nola's Tables to Contemporary Cartographical Data
}

\author{
Alessandra Avella \\ Nicola Pisacane \\ Pasquale Argenziano
}

Abstract

This paper presents the analysis of the sixteenth-century city of Nola through the comparative study of four drawings attached in De Nola treatise by Ambrogio Leone (I5|4), also in comparison with the contemporary city. These drawings are an important graphic-documentary testimony of the city as they not only represent the morphology of the places at the time of their elaboration but also for the methods and techniques used for their execution, therefore offering themselves to considerable evaluations, some of which are collected in this paper.

Starting from graphic and geometric analyzes and metric estimates of the drawings in the Treatise, this research makes use of the methodological approaches of the science of Drawing not only for their critical reinterpretation but also to connect the Renaissance city to traces still existing in the contemporary city. The comparison with the contemporary city is not only aimed at identifying the permanence in the road network but also at some considerations on the representations of Nola by Leone.The drawings, in fact, are different not only for the spatial context represented in them, but also for the methods of representation adopted, sometimes to simulate the three-dimensionality of the orographic reality, in other cases to offer a visual verisimilitude to the architectures and city walls to demonstrate a general control of the designer on the image of the scene represented.

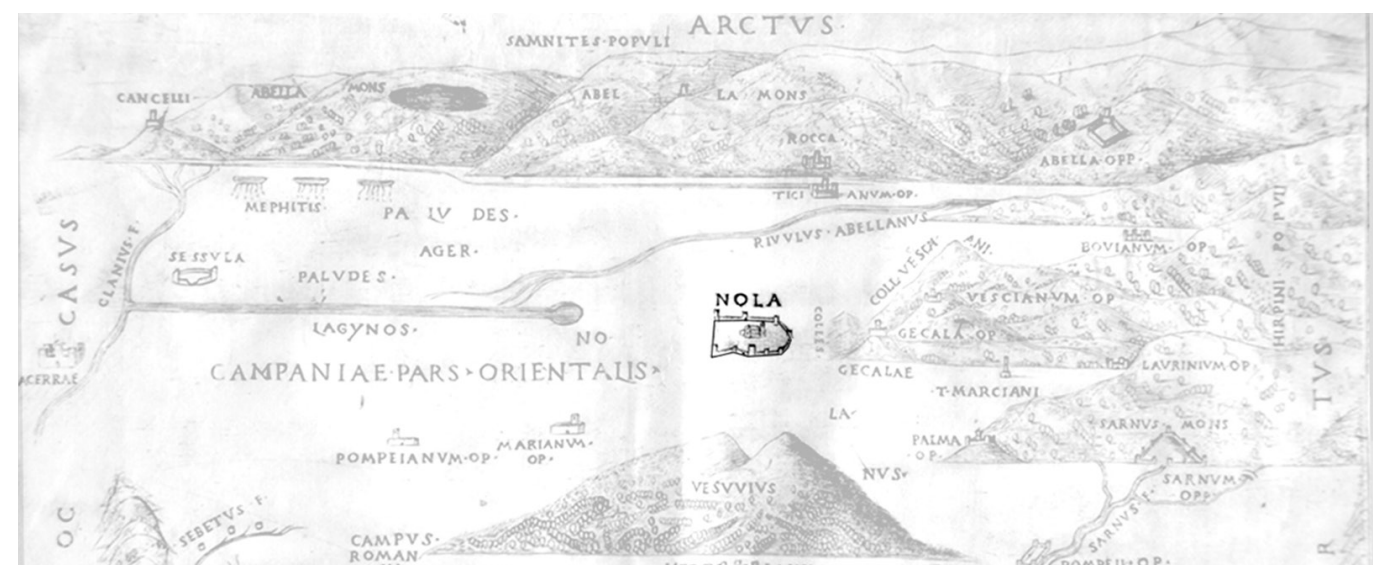




\section{Introduction}

This paper refers the first results of the research conducted within the Research Project funded by Regione Campania and aimed at the study for the safeguard and enhancement of the cultural elements registered on the UNESCO Intangible Heritage List [ I]. Specifically, this research analyzes the drawings attached in De Nola treatise by Ambrogio Leone (15/4) [2] for which an unprecedented reading in the overview of studies, large and multidisciplinary published over the past decades, is proposed deepening the geometric and graphic aspects according to a methodological approach specific to the discipline of Drawing [3]. Leaving aside the bibliographic luck that the Work in Latin has had over the centuries [4], this research proposes a graphic reinterpretation of the hypotheses presented in the critical essays on the iconographies of the Leone's Work published from the early twentieth century [5] until a few years ago by active scholars in various fields of Italian research.

The analysis of all drawings (fig. I) is conducted on one of the copies of the first printed edition preserved in the National Library in Naples [6]. After the first one edited by Leone and probably by Mocetto [7], there are six Latin editions in miscellaneous or monographic volumes [8] and two Italian translations of De Nola [9].

Leone organizes the work into three books through which in order he describes: the Nola territory with a chronological slant and with a wide dissertation on the ancient city; the city coeval with him and its structure with in-depth analysis about the defensive elements, the aristocratic, religious and civil architectural emergencies; and finally, giving the work a touch of color that is rarely found in coeval homologous publications, he describes the uses and customs of his fellow citizens, including the tradition of the 'Festa dei Gigli' [ I 0], from whose study this research draws inspiration.

The edition of I5 I4 published in Venice by Giovanni Rosso da Vercelli, is made up of 64 printed papers, among which there are four out of text tables; these, imprinted on sheets other than the bound ones, were glued to the blank pages suitable for receiving them.

The Ager Nolanus table (Table I) is located to the left of the beginning of Liber Primus (bks. I, I) [ I I]; the Nola Vetus table (Table II) is located to the right page of the end of the De increment et potenti Nolanorum chapter (bks. I, 6); the Figure praesentis urbis Nolae table (Table III) is located to the left of the end of the De figure et in praesentis chapter (bks. II, 2); the Nola Presens table (Table IV) is always located to the left of the beginning of the De muriis, pomoeriis chapter $[\ldots]$ preasentis urbis (bks. II, 7) [I2].

Fig. I. Four drawings from De Nola (edition | 14 ): (top left) Ager Nolanus (called Table I), (bottom left) Nola Vetus (called Table II); (top right) Figura praesentis urbis Nolae (called Table III); (bottom right) Nola presens (called Table IV).

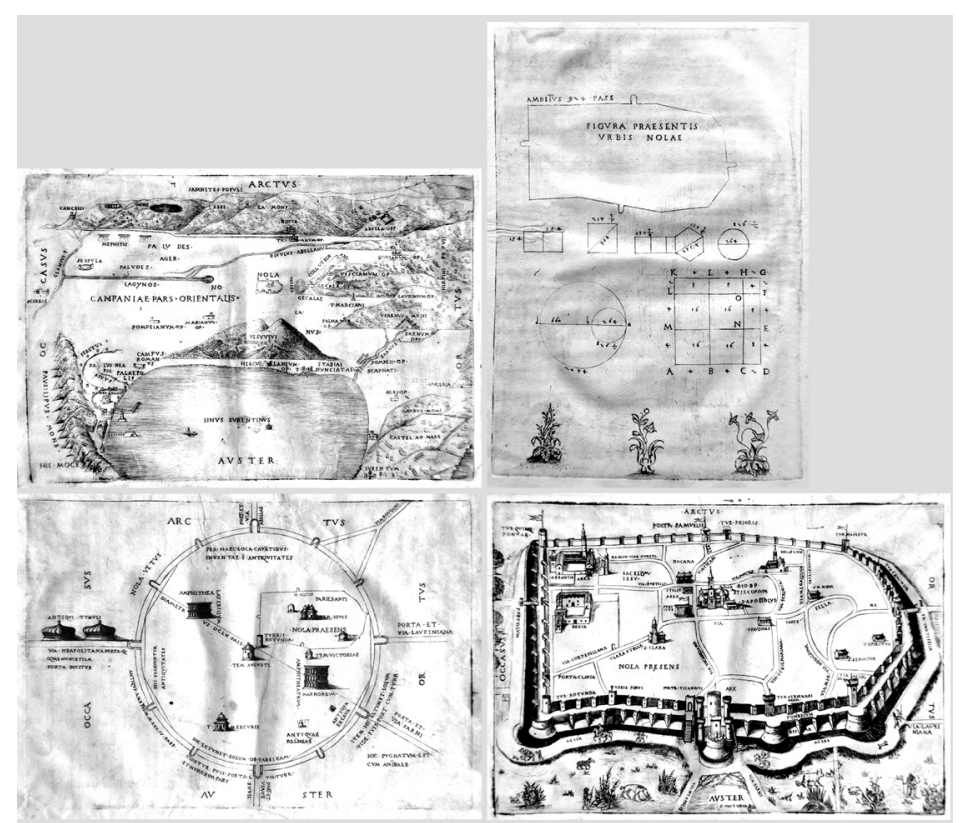


The tables, with the exception of Table III which has a predominantly geometric character, have in common the use of different methods of representation, favoring an accidental representation with a viewpoint in an elevated position [Manzi 1973]. Specifically, Table I -the only one signed by Girolamo Mocetto- has a viewpoint from the Gulf of Naples to the north with alignment in the direction of the western city walls of the city of Nola.

The allusion to three-dimensionality is evident from the representation of the orography. Table II seems to be an icnography of the city walls layout of the ancient city along which the vertical projection of the 12 urban gates is represented reversed in the direction perpendicular to the rays. The architectures represented mainly in orthogonal vertical projection appear iconic of the respective typologies rather than allusive of a verisimilitude with reality. Table IV appears as a scalar focus of Table I on the city, thus deepening a likely representation of the places seen by Leone, both of the defensive system and of the architectures, also enriched by the design of the road network.

These four tables have the common alignment of the western city wall with a constant north-south orientation, regardless of the method of representation adopted and always parallel to the vertical edge of the table, almost as if to be the identifying sign of Nola regardless of the scale of the representation. This direction lies along the vertical median of Tables I and II, while in Table IV it aligns the fortified Citadel with the Cathedral.

All Tables are accompanied by didactic texts in capital characters of variable size in relation to the type of information; once again an exception is Table III in which the alphanumeric dimensioning system is in italics.

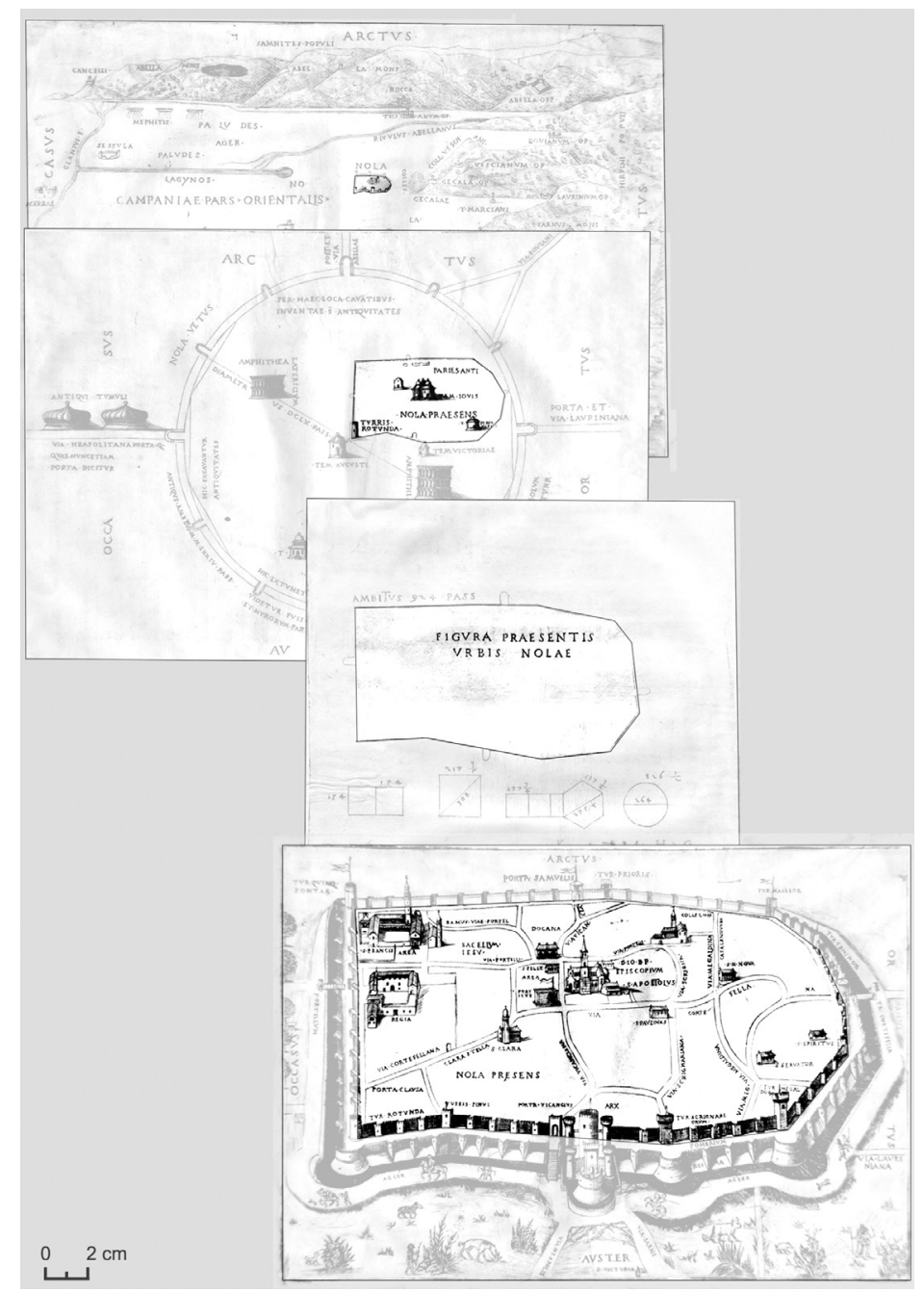




\section{Materials, methods and discussion}

In the context of the main research on the iconographies attached in De Nola treatise, this paper describes the investigative process from the methodology to the testes made on four drawings representing the Renaissance city in relation to the shape of ancient city, its size, its urban system and the main architectures in it. The analysis continued with some critical assessments of the methods of representation, the shape and measurement ratios of each drawings, and the Renaissance city shape, compared with the most up-to-date digital map data on the GIS platform [13].

The research was developed on the drawings printed into the first edition (I5 I4) evidently to trace the original graphic ideas of Leone and Mocetto, who together also edited the book, probably. This choice is linked to lay solid basis for the analysis of the original iconography in comparison with those printed in subsequent editions. The analysis of the iconographies in the Latin editions will be published later. The tables attached to the consulted copy of the

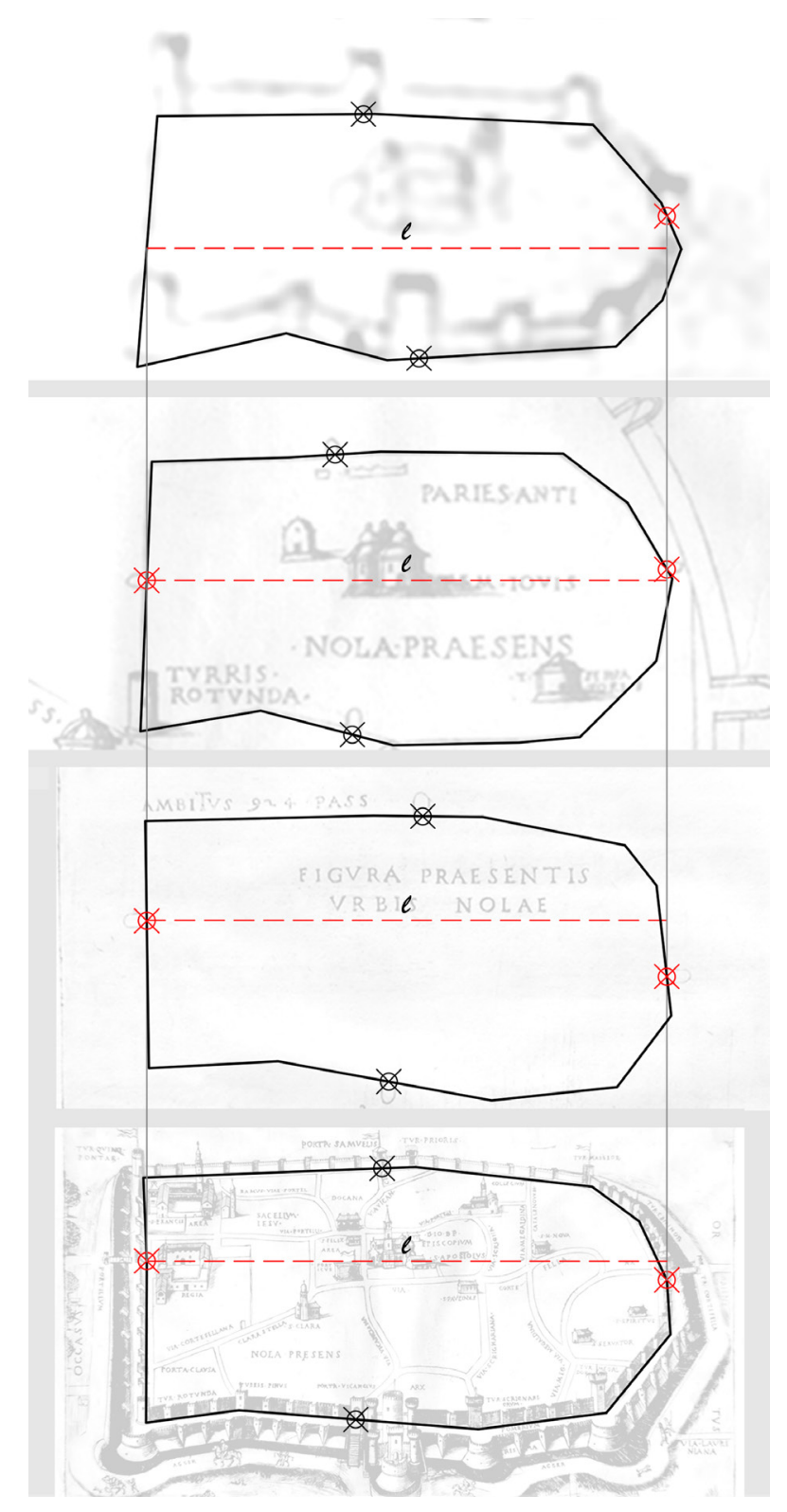


Fig. 4. Table III, identification and Classification of the vertices and urban gates on the polygonal line of the city wall.

Fig. 5. Table IV identification and classification of the vertices and urban gates on the polygonal line of the city wall.
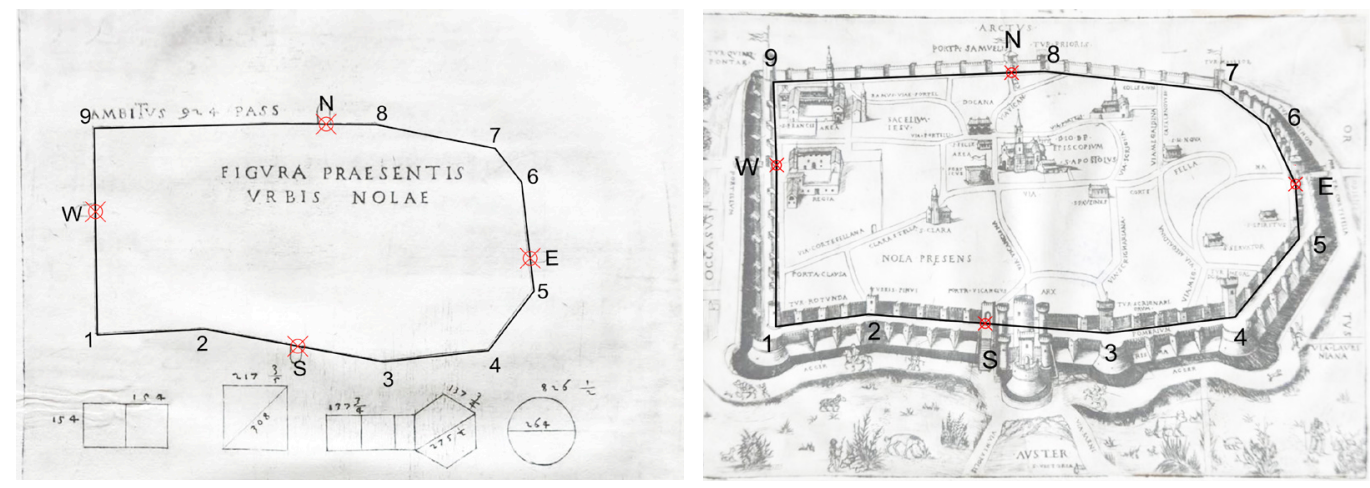

treatise -as is evident- have undergone modifications as result of the environmental conditions, and therefore their physical consistency is not exactly comparable to the original one. In order to fix the consistency of the tables and the beginning of the research, and to solve the close-range photograph aberrations, the digital images capturing was made pulling the originals drawings, a millimetre scale and two chromatic scales (CMYK and gray) together; and also the sheets and the images' frame were measured.

Using in the RDF software [14], the dimensions of the sheets and images imprinted on them were used to orthorectify and scale the photographs to the real size [15]. The chromatic scales were later used to reproduce the real colouring of the iconography in one of the most popular photo-editing software. According to this procedure, graphic analysis is based on photographic 'models' in true shape, size and color of the iconography of I 5 I 4 .

Fig. 6. City path, vertices and urban gates in Table III compared to those
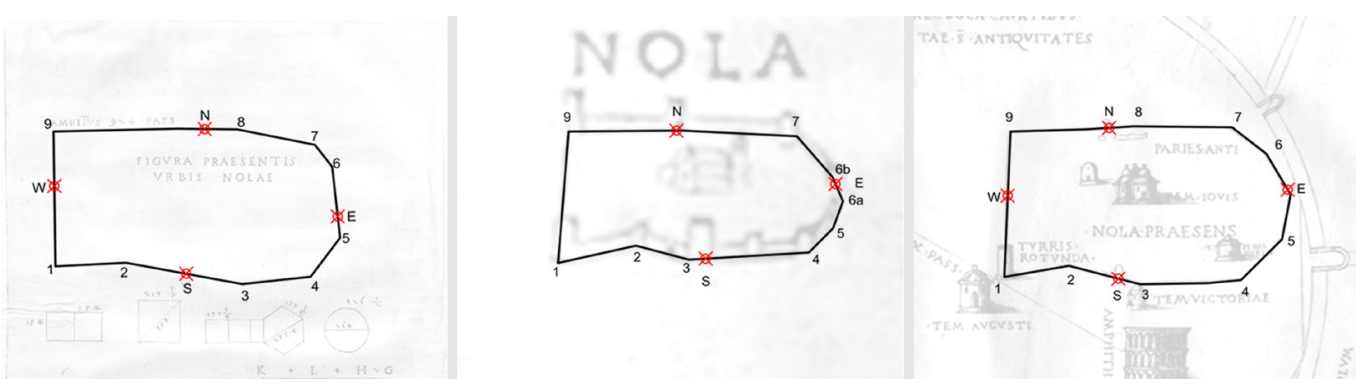

The analysis of the drawings scale-compared (fig. 2) shows that the perimeter -titled by Leone as Figure praesentis urbis Nolae- is the only sign common to them, and therefore subsequent analyses are conducted from this element. To support this choice, there is the hypothesis that Leone had made a geometric survey of the urban perimeter [De Divitiis, Lenzo, Miletti 2018 p. 60], and that Table III is its drawing, also given the geometric and dimensional evaluations, explained into the text [Leone I5|4, bks. II, 2].

This perimeter, is titled as Nola praesens in Table II, and it is well identifiable in the iconic shape drawn in Table I, and it can also be traced back to the inner city wall in Table IV.

The analysis of the drawings in comparison with the perimeter of Table III, shows that the perimeter drawn in Table I is $12 \%$ less, that one in Table II is $50 \%$ less, while that one in Table $\mathrm{IV}$ is $170 \%$ more. 


\section{From four drawings attached in De Nola, to the graphic-geometric analysis of the urban perimeter}

The comparison among the drawings into the raster software environment could not be continued due to excessive discrepancies among the profiles' portions of the wall perimeter. Therefore, the research continued through the vector redrawing of the urban icnography. The positions of the urban gates -according the cardinal directions, as described by Leonewere intercepted along the representative polygons of the urban perimeter. The east-west distance (baseline I) from the west gate has been taken as a common reference to four drawings in order to make it comparable, also because the west gate is the only one still recognisable in the contemporary city and it is evidently represented in Tables II, III and IV; otherwise the drawings would not be overlapping. In Table I the west gate is not drawn and therefore its position has been calculated at a proportional distance to the average of the previous three, along the western wall segment. The I baseline is comparable in these four drawings, being parallel to the representation frameworks, regardless of the adopted representation method. Therefore, these four polygons were scaled relative to it (fig. 3).

As mentioned above, subsequent comparisons take Table III as a reference image, since Leone offers an accurate description of its table even with precise references to the perimeter and the area of the city. The essentiality of this drawing allows to identify nine vertices of the representative polygon of the city, along with four gates, whose vertical projections appear graphically tipped over the plane around the perimeter. The vertex numbering shown in Figure 4 starts from the vertex corresponding to the southwest defensive tower, and proceeds counterclockwise, according to the most widespread geometry convention (fig. 4).

Fig. 7. Comparision between the city wall edge (in orange) from able III, scaled from the length of 924 steps, and the one (in blue) taken from Table IV.The two polygons, keeping the length of the I baseline fixed (east-west distance starting from the west door), are aligned in first hypothesis (top left) in vertex I, then (in the center left) with the two baselines coincident. Evaluating the coordinates luating the coordinates xy system origin xy system originating in point I (bottom left). In the tables on the right, from top to bottom, evaluation of the dimensiona variations in the length of the single segments of city wall and the inner angles of the nine vertices, and the position changes of the individual vertices expressed in steps.
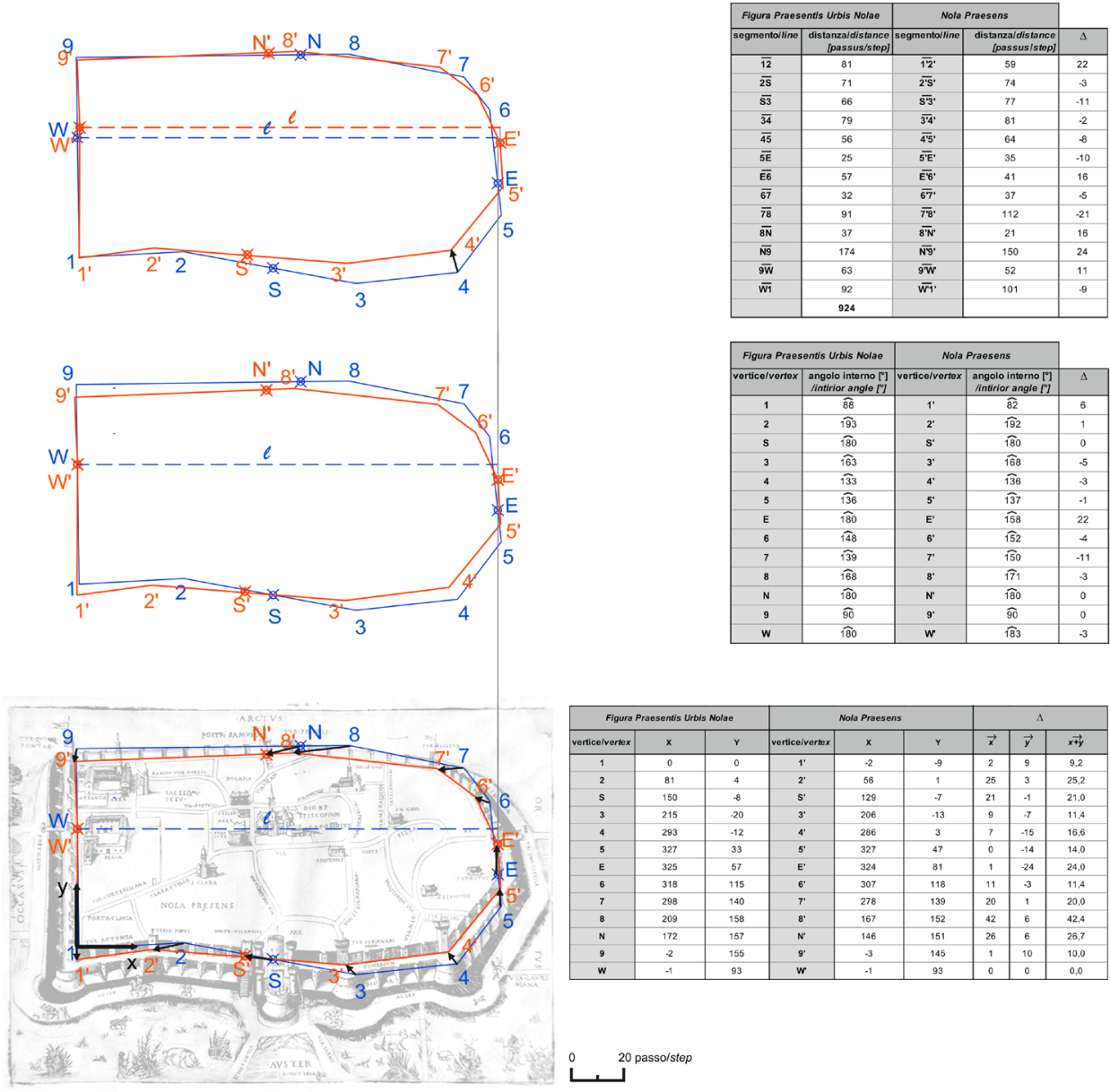
In Table IV (fig. 5), the homologous vertex -corresponding to the defensive towers of the inner wall- are clearly identifiable because the drawing simulates the third dimension, and emerging from the city wall. In this drawing, nine towers -excluding vertex 5-are indicated by the corresponding name. The urban gates have their corresponding title. In addition, the east gate is located on a further vertex of the polygon and it is not on a point in segment $5-6$, as it appears in the previously commented image. The drawing in table IV is done with a three-dimensional allusive method, because the vertical shape of the city wall and of some buildings within it appears. The western wall segment is an exception, because it appears with a depth glimpse, and therefore the draftsman represented the height of the wall in a non-vertical direction.

A similar comparison is possible among Table III and Tables I and II. Nine vertices are always identifiable as shown by comparative analysis. In Table I -the representation of the Renaissance city is placed in the wider territorial context- there are eight vertices along this polygon segment, as the vertices $I$ and 7 are identifiable in two drawings. Along the segment 7-9, vertex 8 is not on the wall perimeter, and also it is not identifiable through the tower's shape, as is possible for other vertices. The towers are represented and recognisable in the drawing, although the graphical scale does not allow for high graphic detail. This simplification - $s$ already commented- has also been carried out for the west gate, where the orientation of the wall in reference to the point of view, leads to a glimpse; the draftsman probably solves this glimpse by erasing this gate.

In Table II, on the other hand, the east gate is located on a further vertex of the polygon, similar to what is in Table IV. Four gates are represented in vertical projection with two different tipping; the western, the northern and eastern gates are overturned outside the perimeter, the fourth one is overturned inwards. The drawing also reproduces the shape of a circular tower at vertex I, that is also the center of the circumference corresponding to the perimeter of the Roman city, according to Leone. The most recent reference (De Divitiis, Lenzo, Miletti 2018 pp. 70) proposes that the surveyor set itself on this corner tower to develop the topographical surveying from one of the eminent points on the city, evidently (fig. 6).

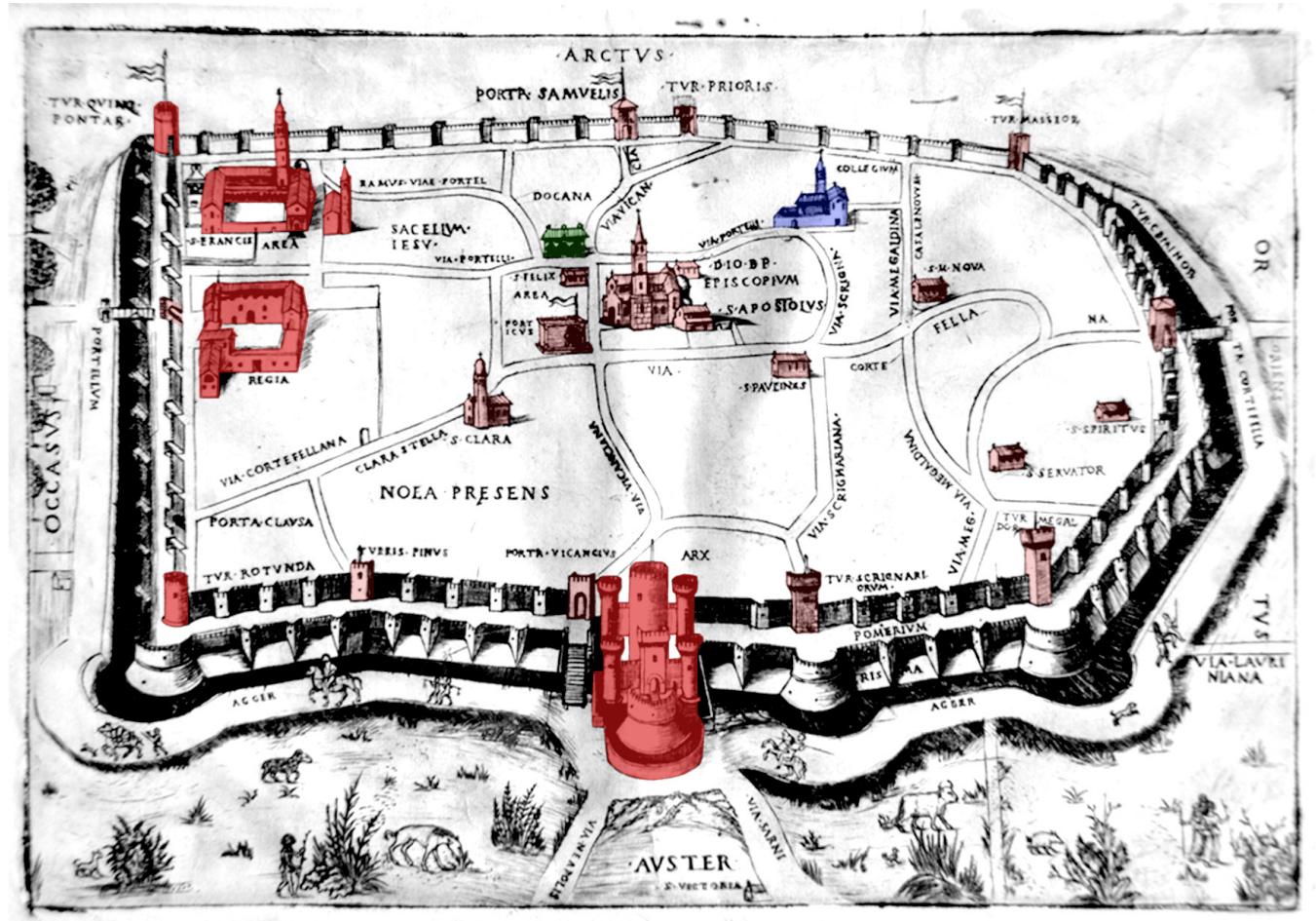




\section{From metric and dimensional assessments to hypotheses about representation methods in the Nola Presens drawing}

The analysis continues with the comparison between Tables III and IV, which are the most significant and detailed for the study of the Renaissance city and of the representation methods, used in the examined drawings. The polygon extracted from Table III was scaled to the size of 924 steps, the only dimensional data, written by Leone. This operation was done in order to assess the actual length of the individual segments of the urban wall among the towers, and among the towers and the gates.

The known distance is the comparison element with Table IV, whose values are used to calculate the variations of the above segments. A similar assessment was made for the inner corners of all polygon vertices and all gates points. Again, angular variation is useful for some assessments.

As already written, the urban perimeters in the drawings are not overlapping, and so a more thorough examination of polygons is conducted according to two hypotheses. The first hypothesis coincides the two polygons in vertex I by aligning them according to segment I-9, considering how rigid the length of the I base line - calculated as 326 steps. The second hypothesis, on the other hand, coincides the baselines of two polygons originating in the west vertex. The comparison is done by referring the positions of the vertices with respect to a pair of orthogonal axes oriented with origin in point I. The choice of this point is linked to the hypothesis that it was the station point of topographical surveying, as already written; it also allows to work most of the positive coordinates. The displacement vector is also calculated for the thirteen vertices, of which nine represent the towers and the remaining are the gates; the calculation is done in the direction of the axes and the ordered, then as a sum vector.

The analysis of these values shows that in the first hypothesis the perimeters, if they are almost overlapping for the entire path between vertices 2 and $\mathrm{E}$, have a rigid translation of about 18 steps in the vertices 3 and 4 . This hypothesis suggests that the draftsman could have deformed the profile to accommodate a pseudo-axonometric projection from southeast, according to an oblique direction of the center of view. The second hypothesis identifies the changes in the position of the vertices such that:

Fig. 9. Georeferentiation in GIS platform of the main points of the road path in Table IV and still recognizable in the contemporary city. Identification of the road. path and 30 points on path and 30 points on (the current orthophoto (top left), identification of the homologous points on Table IV and indication of relocation vectors (top right) and summary table of the pixel coordinates of the historical image and the map coordinates (map system WGS84/ UTM 33N) of the homologous points and their relocations (bottom).
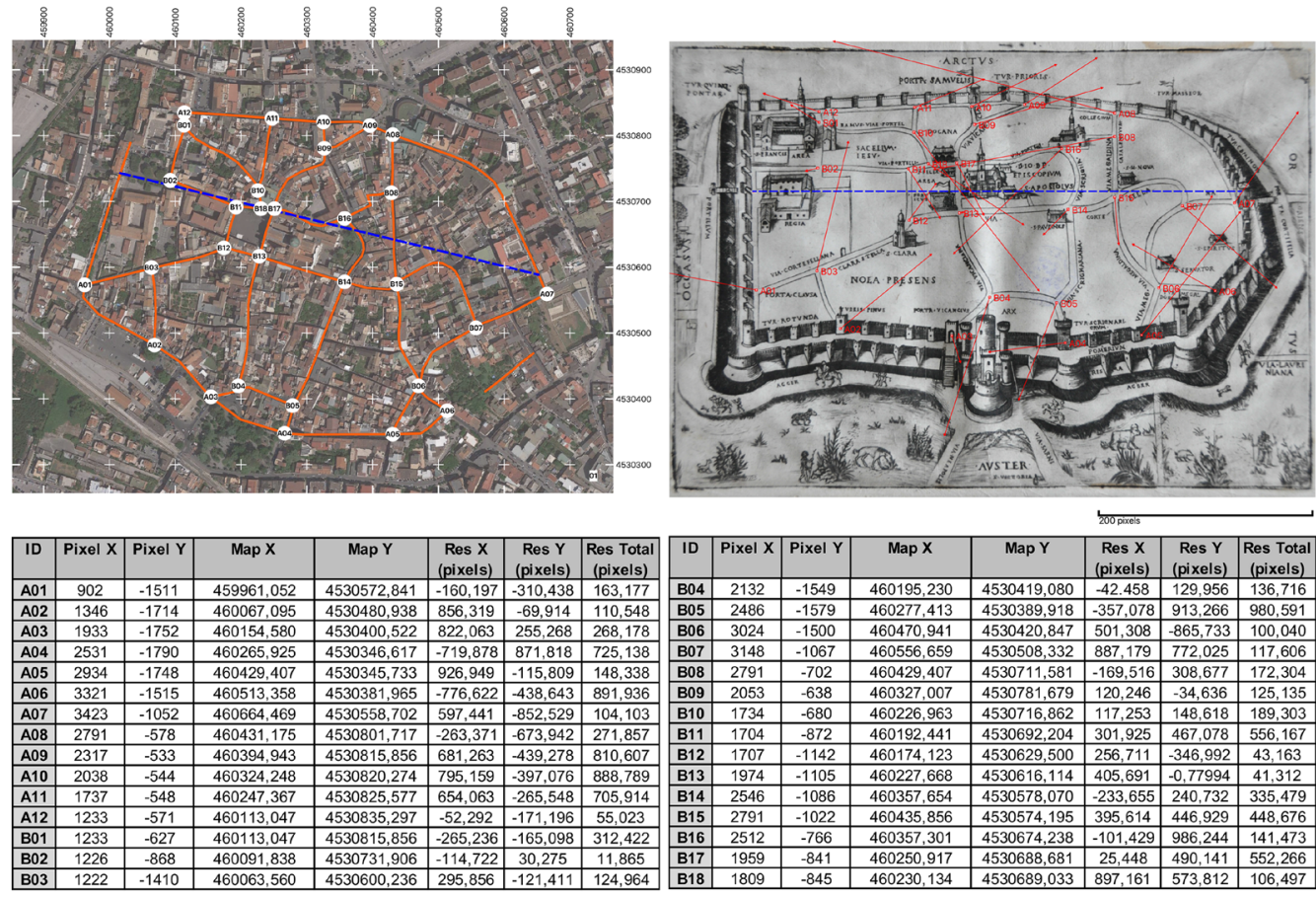
- where the change in displacement along the direction of $x$-axis is greater, the value of the displacement along the direction of $y$-axis is minimal; and vice versa.

- the vertices 9, N, 8 and 3, 4, respectively belonging to the northern and southern section of the city walls, have the minor displacement according to the vertical direction and almost constant value, as well as lower, than that of all the others.

- the movements value, with the exception of vertex 8, have a variation between 9.2 and 26.7 steps (fig. 7).

The aforementioned considerations assume that the image in Table IV is represented in a pseudo-axonometric view, not only for the drawing of the elevation in architectural elements, but also for the constant reduction of the dimensions in depth, which suggest an orthogonal position of the axonometric projection point of view, as evidently observed from the image of the Castle, the Palace and 'San Francesco' area.
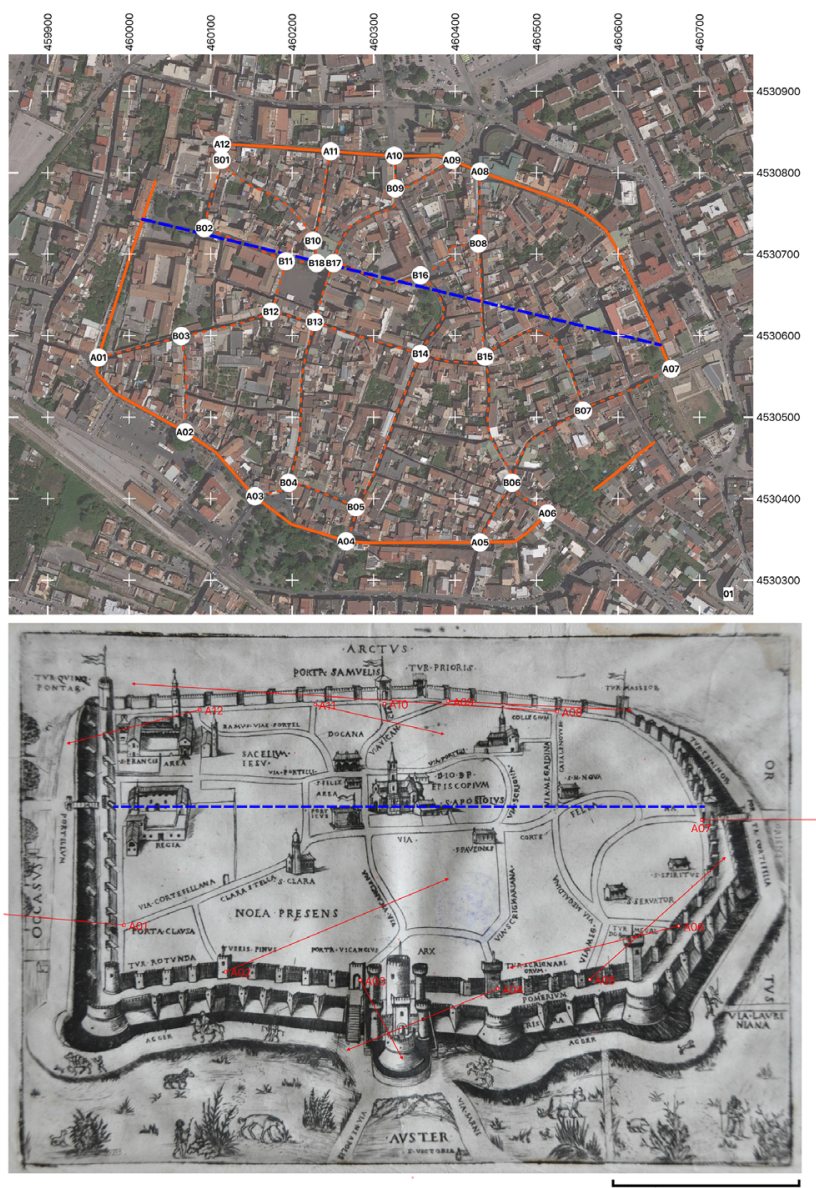

\begin{tabular}{|l|c|c|c|c|c|c|c|}
\hline ID & Pixel X & Pixel Y & Map X & Map Y & $\begin{array}{c}\text { Res X } \\
\text { (pixels) }\end{array}$ & $\begin{array}{c}\text { Res Y } \\
\text { (pixels) }\end{array}$ & $\begin{array}{c}\text { Res Total } \\
\text { (pixels) }\end{array}$ \\
\hline A01 & 902 & -1511 & 459961,052 & 4530572,841 & $-110,707$ & $-101,244$ & 111,169 \\
\hline A02 & 1346 & -1714 & 460067,095 & 4530480,938 & 118,952 & $-497,587$ & 128,94 \\
\hline A03 & 1933 & -1752 & 460154,58 & 4530400,522 & 225,432 & 421,825 & 478,284 \\
\hline A04 & 2531 & -1790 & 460265,925 & 4530346,617 & $-80,483$ & 328,623 & 869,336 \\
\hline A05 & 2934 & -1748 & 460429,407 & 4530345,733 & 725,372 & $-65,407$ & 976,715 \\
\hline A06 & 3321 & -1515 & 460513,358 & 4530381,965 & $-901,669$ & 223,516 & 92,896 \\
\hline A07 & 3423 & -1052 & 460664,469 & 4530558,702 & 961,215 & $-0,22677$ & 961,218 \\
\hline A08 & 2791 & -578 & 460431,175 & 4530801,717 & $-227,33$ & $-148,496$ & 227,814 \\
\hline A09 & 2317 & -533 & 460394,943 & 4530815,856 & 979,477 & 491,833 & 980,711 \\
\hline A10 & 2038 & -544 & 460324,248 & 4530820,274 & 964,157 & 628,821 & 966,205 \\
\hline A11 & 1737 & -548 & 460247,367 & 4530825,577 & 686,935 & 159,102 & 705,119 \\
\hline A12 & 1233 & -571 & 460113,047 & 4530835,297 & $-709,393$ & 180,915 & 732,098 \\
\hline
\end{tabular}




\section{Continuity between Renaissance city and contemporary one}

A common element in the whole scene is offered by the allusive representation of three-dimensionality. The draftsman associates to a front view a side glimpse of hard-to-control. In fact, it can be seen that from west to east the glimpse of the buildings and some sections of city walls is parallel by sectors, changing the direction of dimensions in depth. Such graphic artifice seems to simulate the dynamism of the projection center of view. It can be assumed that rather than a pseudo-perspective representation, the image combines multiple axonometric projections. To an approach that testifies the general control of the draftsman on the scene, it is to highlight the drawing of the western city wall with a strongly glimpsed orientation, which is solved with a constant rotation of the direction of the vertical edges. This graphic anomaly contradicts with the correct drawing of the heights of the towers and 'baloardetti' [16] in the 4'-7' section (from the 'Torre Megaldor' to the 'Torre Masseor'). The analysis has been deepened by classifying the buildings represented within the city wall and the towers according to the formal assonance to drawing methods (parallel and central projections) to further confirmation of a dynamic point of view. Most of the buildings in the west part appear in orthogonal pseudo-axonometric projection, unlike those drawn in the other part of the city that allude to the use of oblique pseudo-axonometric projection. The exception is the representation of the 'Collegium' building appearing in al perspective (fig. 8).

The small glimpses of dimensions in depth between the profile in Table III and the one in Table IV (fig. 7) suggest that the road path inside the city wall in the Table IV may be the output of a metric survey. Assuming this hypothesis in trueness, through GIS has been georeferenced with control points on the road still recognizable in the contemporary layout. Knowing the resolution of the orthorectified image, it has been scaled to the size in steps of the 'l' baseline (326 steps) corresponding to 2580 pixels. Therefore the pixel has a size of 0.13 steps.

The 9 towers and city gates, excluding the west one, are not recognizable in the contemporary city so it has been identified the 12 points of intersection between the road along the city wall and the roads to it converge. Therefore, the georeferentiation has been first carried out through 30 homologous points, of which the remaining 18 are crossing points between roads, still recognizable in the comparison between the drawing and the current orthophoto (fig. 9). Subsequently, a similar operation was conducted through only 12 vertices along the city wall (fig. I0). Leaving aside the outcome of the graphic deformation of the image (Table IV) which produces a significant variation of the shape that detracts from numerical assessments, the critical evaluation of georeferentiation can be made by estimating the variations between the pixel coordinates of the historical image and the map coordinates of the georeferenced point. These values, expressed in pixels and therefore attributable to the size in steps, with reference to the aforementioned dimensional match, show greater deviation values for the internal points exemplifying the road crossings than those on the city wall.

\section{Conclusions}

The results of the comparative analysis after georeferentiation confirm that the draftsman has drawn up Table IV starting from the path of the city wall perimeter following a metric survey, rather than from the complete plan of the road and the main buildings. It is no coincidence that the only metric dimension in the work is related to the perimeter of 924 steps of the city wall.

A further assessment, referring to the comparison described above, relates to the position and orientation of the baseline I as outcome of the georeferentiation. This segment, while in the tables has a constant orientation $90^{\circ} \mathrm{N}$, in the actual topography is rotated $14^{\circ} \mathrm{compa}-$ red to the previous one. It is easy to gather that Leone forced the horizontal arrangement of the city gates according to the orientation of the paper frame. 
These results open up further and unexplored possible investigative scenarios which, starting from considerations supported by the graphic applications of the drawing's own tools and methods, may provide additional elements to assess whether the representations are really refunds of a metric survey and therefore the result of a successful activity of acquisition of the metric data, as reported in the literature. If so, the research will delve into how these detection operations were conducted and with what tools in use in Leone's time, as well as estimating the accuracy of the return from the captured data. The GIS platform, already assessed as suitable for the geometric comparison operations described above, will be able to be confirmed as a useful aid for further analyses that are proposed regarding the calculation of the possible error of data acquisition and units used by Leone with today's metric systems [17].

\section{Notes}

[I] 'Gigli di Nola' celebration are included, together with the 'Nostra Signora della Santa Lettera' of Palmi, the 'Discesa dei Candelieri' of Sassari and the 'Macchina di Santa Rosa' of Viterbo, in the "Network of large Italian shoulder machines", inscribed since 2013 in the UNESCO Intangible Heritage List.

[2] Ambrogio Leone (Nola $1458 \mathrm{ca}$ - Venice 1525) is a singular person of Italian Renaissance culture, connecting the environments of the Neapolitan scholars, the Venetian ones and those from beyond the Alps who frequented their respective capitals. He obtained his doctorate in medicine and philosophy in Padova at the age of 26 and returned to Nola he practiced the medical profession in his home outside the city, which became a meeting place for eminent men of culture, including Giovanni Pontano, a well-known and influential humanist and politician, and Aurelio Bienato from Milano, reader of rhetoric at the University of Naples. At the beginning of the new century, Leone moved first to Padua and then to Venice practicing his profession in the San Marco district and occasionally returning to Nola until the flood of I 504 which profoundly marked the fate of the Campania city. In Venice he had the opportunity to distinguish himself among the circles of the lagoon culture, so much so that he had the opportunity to forge friendships with Aldo Manuzio, entering his circle of scholars, known as "dei Filelleni". This is particularly important for De Nola's cultural location and graphic evaluations. The constant attendance of this cultural circle allowed him to get to know Erasmus of Rotterdam with whom he remained in contact beyond the period of residence of the Dutchman in the Lagoon, as documented by two letters sent between 1518 and 19.

In January 1510, the funeral celebrations of Niccolò Orsini - count of Nola and general captain of the Venetian troops in the war of the Lega di Cambrai (I508-|5|6) - were crucial for the realization of De Nola, because on that occasion he certainly had the opportunity of meeting (if he had not previously done so) Girolamo Mocetto. Leone attended the funeral because Nolano and friend of the Orsini family, Mocetto at that time lives in Venice and was active in the Dominican church of SS. Giovanni and Paolo of Venice because he is engaged in the decoration of the painted window of the right transept, in which he will insert, among others, the figure of the leader Orsini as a sign of commemoration [Spruit 2005+.

[3] See:Vagnetti 1973; Pagnano 2003; Zerlenga 2004.

[4] Carillo 1996;Valerio 1998; De Divitiis, Lenzo, Miletti 2018.

[5] It is useful to the framework of this paper does not list all the publications published and consulted, referring to the specific references in other notes. The review of the complete bibliography is in: De Divitiis, Lenzo, Miletti 2018.

[6] Shelfmark SQ.LVI.D. 16

[7] Girolamo Mocetto (Murano $1470 \mathrm{ca}$ - 153 I ca), descendant of a family of glassmakers, is more active in painting and engraving than the family tradition. Although there is no certain information on his artistic training, three known hypotheses allow him to be well qualified: in the work Vite, Vasari indicates him as a pupil of Giovanni Bellini; in more recent times, others scholars support the hypothesis of frequenting Alvise Vivarini's workshop in Murano or of belonging to the circle of Andrea Mantegna, from whom he would have learned the burin engraving art. Tagliaferro (20I I).

[8] See: De Divitiis, Lenzo, Miletti 20 I8, p. 5.

[9] See: Leone 1934; Leone 1997.

[10] See: Leone I5|4, bks. III, 7.

[I I] This abbreviation refers to the reference to the Book in cardinal numbering and that to the Chapters of the work in Arabic numbering (ISO 690: 1987).

[12] The catalog of drawings published during the eighteenth century is in: de Seta, Buccaro 2006, pp. $273,274$.

[13] The research on the drawing of De Nola integrates the studies on Italian landscape painting and cartography of the Sixteenth Century. The latest essays read on these topics are: de Seta, Buccaro 2006; and the monographic issue of DisegnareCon on Drawing the Territory and the Landscape (vol.12 no.22, 2019) edited by Pilar Chìs Navarro and Lia Maria Papa; in this journal, it's interesting Aguilar-Camacho, Joaquín, Granado-Castro, Gabriel and Barrera-Vera, José Antonio, Urban mapping of Cadiz during the War of Spanish Succession: a paradigm of the cartography enterprise undertaken by the Military Engineer Corps, pp. I- I 3.

[ I 4] The RDF software is open thanks to CIRCE Photogrammetry Laboratory at IUAV University in Venice:<http://www.iuav. it/SISTEMA-DE/Laboratori2/cosa-offri/software/index.htm>. 
[15] The frames dimesions of the Tables examined are in order: $\mathrm{cm} 28 \times 19.3 ; \mathrm{cm} 27.7 \times 19.2 ; \mathrm{cm}$ 19.7×28.1; 27.6x19.1; sheets have no watermark.

[16] Reinforcing prismatic buttresses in the city wall between two towers: Sardi 16 18, p. 40.

[17] The authors designed the research and shared its methodology and contents. In particular, the paragraph entitled "From four drawings attached in De Nola, to the graphic-geometric analysis of the urban perimeter" is edited by Pasquale Argenziano, the paragraph entitled "From metric and dimensional assessments to hypotheses about representation methods in the Nola Presens drawing" is edited by Alessandra Avella, while the paragraph entitled "Continuity between Renaissance city and contemporary one" is edited by Nicola Pisacane. Introduction and conclusions are edited by all the authors. Figures, except for archive images, are edited by authors.

\section{References}

Carillo Saverio (1996). Alcune osservazioni sulla pianta della città allegata al De Nola di Ambrogio Leone. In:Toscano Tobia R. Nola e il suo territorio dalla fine del Medio Evo al XVII secolo. Momenti di storia culturale e artistica. Castellammare di Stabia: Ager Nolanus. p. 25-44.

De Divitiis Bianca, Lenzo Fulvio, Miletti Lorenzo (a cura di). (2018). Ambrogio Leone's De Nola, Venice 15I4. Humanism and Antiquarian Culture in Renaissance Southern Italy. Leiden, Boston: Brill.

De Seta Cesare, Buccaro Alfredo (a cura di). (2006). Iconografia delle città in Campania. Napoli e i centri della provincia. Napoli: Electa Napoli.

Leone Ambrogio (15|4). De Nola. Opusculum distinctum, plenum, clarum, doctum, pulchrum, verum, grave, varium, et utile. Venezia: Giovanni Rosso.

Leone Ambrogio (1934). Nola (la terra natia). Napoli:Tipografia Torella.

Leone Ambrogio (1997). Nola. Napoli; Istituto Grafico Editoriale Italiano.

Manzi Pietro (1973). Alcuni documenti di cartografia nolana ovvero: Ambrogio Leone e Gerolamo Moceto. In L'Universo. Vol. LIII, no. 4, p. 81 I-8I8.

Pagnano Giuseppe (2003). Presentazione. In Ikhnos. Analisi grafica e storia della rappresentazione. Vol. I, p. 7- I 0.

Sardi Pietro (16|8). Corona imperiale dell' architettura militare.Venezia: Barezzo Barezzi.

Spriut Leendert (2005). Leone, Ambrigio. In Dizionario Biografico degli Italiani, ad vocem. Treccani: <http://www.treccani.it/ enciclopedia/ambrogio-leone_(Dizionario-Biografico)/>.

Tagliaferro Giorgio, Mocetto Girolamo (20 I I). In Dizionario Biografico degli Italiani. Ad vocem. Treccani.

Vagnetti Luigi (1973). L'architetto nella storia di Occidente. Firenze:Teorema.

Valerio Vladimiro (1998). Piante e vedute di Napoli dal 1486 al 1599. L'origine dell'iconografia urbana europea. Napoli: Electa Napoli.

Zerlenga Ornella (2004). II disegno della città. Napoli rappresentata in Pianta e Veduta. In Ikhnos. Analisi grafica e storia della rappresentazione. Vol. II, p. I| -34.

\section{Authors}

Alessandra Avella, Università della Campania “Luigi Vanvitelli”, alessandra.avella@unicampania.it,

Nicola Pisacane, Università della Campania “Luigi Vanvitelli”, nicola.pisacane@unicampania.it,

Pasquale Argenziano, Università della Campania "Luigi Vanvitelli", pasquale.argenziano@unicampania.it

To cite this chapter: Avella Alessandra, Pisacane Nicola, Argenziano Pasquale (2020). Il disegno della città rinascimentale dalle illustrazioni del De Nola ai dati cartografici contemporanei/ The Drawing of the Renaissance city from De Nola's tables to contemporary cartographical data. In Arena A., Arena M., Brandolino R.G., Colistra D., Ginex G., Mediati D., Nucifora S., Raffa P. (a cura di). Connettere. Un disegno per annodare e tessere. Atti del $42^{\circ}$ Convegno Internazionale dei Docenti delle Discipline della Rappresentazione/Connecting. Drawing for weaving relationships. Proceedings of the 42th International Conference of Representation Disciplines Teachers. Milano: FrancoAngeli, pp. I598- 1621 . 